\title{
Phase-field modeling of domain evolution in ferroelectric materials in the presence of defects
}

\author{
Patrick Fedeli $^{1}$, Marc Kamlah ${ }^{2}$ and Attilio Frangi ${ }^{1}$ \\ ${ }^{1}$ Department of Civil and Environmental Engineering, Politecnico di Milano, \\ Milano, Italy \\ ${ }^{2}$ Institute for Applied Materials, Karlsruhe Institute of Technology, Karlsruhe, \\ Germany \\ E-mail: patrick.fedeli@polimi.it
}

\begin{abstract}
The properties of ferroelectric devices are strongly influenced, besides crystallographic features, by defects in the material. To study this effect, a fully coupled electromechanical phase-field model for 2D ferroelectric volume elements has been developed and implemented in a Finite Element code. Different kinds of defects were considered: holes, point charges and polarization pinning in single crystals, as well as grain boundaries in polycrystals, without and with additional dielectric interphase. The impact of the various types of defects on the domain configuration and the overall coercive field strength is discussed in detail. It can be seen that defects lead to nucleation of new domains. Compared to the energy barrier for switching in an ideal single crystal, the overall coercive field strength is significantly reduced towards realistic values as they are found in ferroelectric devices. Also the simulated hysteresis loops show a more realistic shape in the presence of defects.
\end{abstract}

Keywords: phase-field modeling, microstructural defects, polycrystalline ferroelectric

Submitted to: Smart Mater. Struct. 


\section{Introduction}

Piezoelectricity has become very popular in innovative technologies and has been utilized in a wide range of industrial applications [1, 2], including sensors, actuators, resonators, capacitors, transducers, energy harvesters, non-volatile FeRAM. Most of available products are based on ferroelectric materials $[3,4,5,6$, 7] since they exhibit a large piezoelectric effect. One of the most prominent materials of this class is the Lead Zirconate Titanate $\left(\mathrm{Pb}\left(\mathrm{Zr}_{1-x} \mathrm{Ti}_{x}\right) \mathrm{O}_{3}\right.$, PZT), a solid solution of ferroelectric $\mathrm{PbTiO}_{3}$ and antiferroelectric $\mathrm{PbZrO}_{3}$, which is available in the form of a polycrystal material. Ferroelectric ceramics have been widely employed as a bulk material, but in recent years they have been also utilized in the form of thin films in an increasing number of MEMS applications $[8,9]$. including print heads for inkjet printers [10], ultrasonic transducers for acoustic applications [11] as well as energy harvesting devices [12].

In general, ferroelectric materials present a spontaneous electrical polarization below a certain temperature, namely the Curie temperature $T_{C}$. This is associated with a paraelectric-ferroelectric phase transition, which consists in the separation of the centers of positive and negative charges. Typically ferroelectric materials are divided in domains, i.e. regions with uniform polarization, separated by interfaces called domain walls. Their appearance is associated with the minimization of the free energy when the material undergoes the phase transition from the high-temperature symmetric phase to the low-temperature phases. Domains can be reoriented by applying an external electrical or stress field which can induce a switching between different metastable states. The reorientation process leads to microstructural evolution which is the origin of the macroscopic non-linear electromechanical behaviour of ferroelectric materials. The capability to orient the electrical polarization, randomly distributed in these materials, into a desired direction is known as poling process. Poling is fundamental in turning inert ceramics $[13,14]$ and thin-film materials $[15,16]$ into electromechanically active materials. Therefore it is of paramount importance, in design and optimization of piezoelectric devices for industrial applications [17], to be able to characterize the coercive field strength, i.e. the magnitude of the electric field required to switch the global polarization, and the remnant polarization value, i.e. the residual polarization when a zero electric field is applied. More in general one is interested in the whole hysteresis loop of the polarization versus the applied electric field $(P \vee s E)$.

Firstly, as evidenced in [18], the shape of the hysteresis loop is strongly affected by the type of material considered, whether single- or poly-crystal. Secondly, a number of contributions [19, 20, 21] have proven that the occurrence of microstructural defects such as oxygen vacancies, space charges, dislocations, grain boundaries and voids arising from fabrication processes can dramatically change the material behavior. For instance, they have an impact on the coercive field strength, which is typically orders of magnitude higher in a single crystal than in real ferroelectric ceramics. Moreover, defects might be responsible for the experimentally observed fatigue and aging of ferroelectrics [22, 23], i.e. the degradation of the material during electrical loading or in time even in the absence of an external loading. The aging phenomenon results in a pinching of the polarization hysteresis loop (see figure 1(a)), whereas fatigue leads to a reduction of the hysteresis cycle (see figure 1(b)).

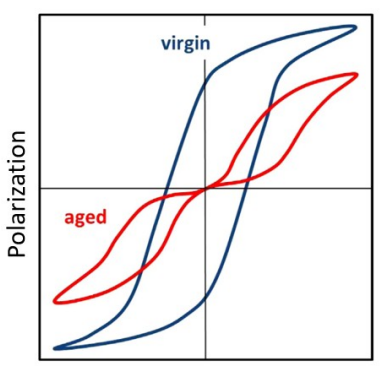

a)

Electric field

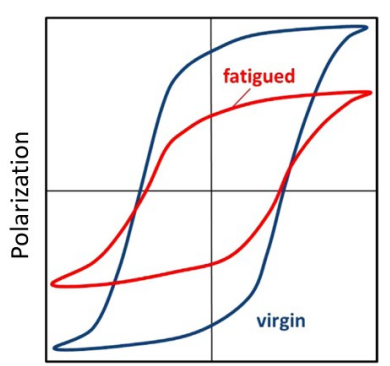

b)

Electric field
Figure 1: Hysteresis loops of the polarization versus the applied electric field for: a) aged and b) fatigued ferroelectric [23].

The linear theory of piezoelectricity [2] is clearly not sufficient to predict the polarization distribution in the material. To understand material properties, one of the key issues is to develop a modelling capability to predict the microstructure evolution, the relationship between microstructure and macroscopic properties, and the impact of microstructural changes on the material response to applied fields. There are many theoretical studies on ferroelectric domain switching and the related non-linear electromechanical behaviour $[24,25,26]$ and a critical literature review has been 
recently published in [27]. The different approaches can be classified into three types, based on the scale of their applicability: macroscopic, mesoscopic and atomic-level methods.

At the macroscopic scale, several phenomenological models have been proposed $[28,29]$ in which the total polarization in the material is divided into a reversible and an irreversible part, the latter being governed by some plasticity-like constitutive law.

At the mesoscopic level, on the contrary micromechanical [30, 31, 32] and phase field models $[33,34,35,36,37,38]$ compute the irreversible part of polarization by simulating the process of domain switching.

At the atomic-level, computational models for ferroelectrics include first-principle methods [39] and molecular dynamics $[40,41,42,43,44,45]$. Because of their computational complexity, these techniques can address small representative volumes in the order of few tens of nanometers. In the context of a multiscale modeling approach like in [46, 47], molecular dynamics can be ideally used to calibrate material parameters for numerical models at larger scales.

In this work we place ourselves at the mesoscale and investigate the behaviour of piezo thin films using the phase-field method (PFM). Since the seminal contribution by Cahn and Hilliard [48], the PFM has emerged as one of the preferred tools to model the evolution of phases and micro-structures in materials (see [33]). It has been recently applied to investigate the non-linear response of ferroelectric single crystals, polycrystals $[49,50,51]$ and thin films $[52,53,54,55,56,57]$ and also to address the interaction between the domain evolution and microstructural defects $[58,59,60,61,62]$. Following $[54,34,37,38]$, we have developed a numerical tool based on the Finite Elements (described in Section 3) with the aim of investigating specific types of defects. Indeed, in Section 4 we simulate specimens including voids, charged point defects, regions with polarization pinning, multigrains with finite dimensional interfaces. For each of these cases we investigate primarily the effect on the global hysteresis loop and show how the response of a perfect crystal is altered towards the observed behaviour of a realistic thin film.

\section{Phase-field model for ferroelectrics}

In accordance with the Landau-Devonshire theory of ferroelectrics [63], the free energy density $\psi$ for a single crystal is assumed to depend on the polarization $\mathbf{P}$, its gradient $\nabla \mathbf{P}$, the small strain tensor $\boldsymbol{\varepsilon}$ and the electric displacement $\mathbf{D}[54,34,37,38]$ :

$$
\begin{aligned}
\psi(\mathbf{P}, \nabla \mathbf{P}, \boldsymbol{\varepsilon}, \mathbf{D})= & \psi_{\mathrm{L}}(\mathbf{P})+\psi_{\text {grad }}(\nabla \mathbf{P}) \\
& +\psi_{\text {mech }}(\mathbf{P}, \boldsymbol{\varepsilon})+\psi_{\text {elec }}(\mathbf{P}, \mathbf{D}) .
\end{aligned}
$$

In what follows, we refer to the free energy density for lead-titanate $\left(\mathrm{PbTiO}_{3}\right)$, which is a ferroelectric material with cubic symmetry, widely discussed in the literature (see, e.g., $[54,55,56,57,59]$ and $[34,61,51,64])$.

The first term in equation (1) denotes the Landau energy density

$$
\begin{aligned}
\psi_{\mathrm{L}}(\mathbf{P})= & \alpha_{1}\left(P_{1}^{2}+P_{2}^{2}+P_{3}^{2}\right)+\alpha_{11}\left(P_{1}^{4}+P_{2}^{4}+P_{3}^{4}\right) \\
& +\alpha_{12}\left(P_{1}^{2} P_{2}^{2}+P_{1}^{2} P_{3}^{2}+P_{2}^{2} P_{3}^{2}\right) \\
& +\alpha_{111}\left(P_{1}^{6}+P_{2}^{6}+P_{3}^{6}\right) \\
& +\alpha_{112}\left(P_{1}^{4}\left(P_{2}^{2}+P_{3}^{2}\right)+P_{2}^{4}\left(P_{1}^{2}+P_{3}^{2}\right)\right. \\
& \left.+P_{3}^{4}\left(P_{1}^{2}+P_{2}^{2}\right)\right)+\alpha_{123}\left(P_{1}^{2} P_{2}^{2} P_{3}^{2}\right)
\end{aligned}
$$

a non-convex functional with minima corresponding to the spontaneous polarization states (SPS) $\mathbf{P}^{s}$. Indeed, an isolated idealized crystal below the Curie temperature $T_{C}$ has several SPS in which atoms find a stable equilibrium with microscopic displacements relative to the lattice. For instance, a tetragonal ferroelectric has six equivalent SPS along the $<100>$ axes.

Regions of homogeneous polarization are called domains and are separated by domain walls. In phase-field models, the domain wall is modelled as a diffuse interface with a finite thickness, in which the polarization changes continuously. The second term $\psi_{\text {grad }}(\nabla \mathbf{P})$ in equation (1) favours the formation of large domains by penalizing the excessive spread of interfaces and fixes the domain wall thickness. In our case we admit a simple "isotropic" formulation with

$$
\begin{aligned}
\psi_{\text {grad }}(\nabla \mathbf{P})= & \frac{1}{2} G_{11}\left(P_{1,1}^{2}+P_{1,2}^{2}+P_{1,3}^{2}+P_{2,1}^{2}\right. \\
& \left.+P_{2,2}^{2}+P_{2,3}^{2}+P_{3,1}^{2}+P_{3,2}^{2}+P_{3,3}^{2}\right) .
\end{aligned}
$$

The third term in equation (1) refers to mechanical energy

$$
\begin{aligned}
\psi_{\text {mech }}(\mathbf{P}, \varepsilon)= & \frac{1}{2} C_{11}\left(e_{11}^{2}+e_{22}^{2}+e_{33}^{2}\right) \\
& +C_{12}\left(e_{11} e_{22}+e_{11} e_{33}+e_{22} e_{33}\right) \\
& +2 C_{44}\left(e_{12}^{2}+e_{13}^{2}+e_{23}^{2}\right)
\end{aligned}
$$

where $C_{i j}$ are the coefficients of the stiffness tensor $\mathcal{C}$ for a material with cubic symmetry, and $\mathbf{e}=\boldsymbol{\varepsilon}-\boldsymbol{\varepsilon}^{s}$ is the elastic strain, with $\varepsilon^{s}$ spontaneous strains caused by the polarization field

$$
\begin{array}{ll}
\varepsilon_{11}^{s}=Q_{11} P_{1}^{2}+Q_{12}\left(P_{2}^{2}+P_{3}^{2}\right), & \varepsilon_{12}^{s}=Q_{44} P_{1} P_{2}, \\
\varepsilon_{22}^{s}=Q_{11} P_{2}^{2}+Q_{12}\left(P_{1}^{2}+P_{3}^{2}\right), & \varepsilon_{23}^{s}=Q_{44} P_{2} P_{3}, \\
\varepsilon_{33}^{s}=Q_{11} P_{3}^{2}+Q_{12}\left(P_{1}^{2}+P_{2}^{2}\right), & \varepsilon_{13}^{s}=Q_{44} P_{1} P_{3},
\end{array}
$$

where $Q_{i j}$ are the electrostrictive coefficients. Equation (5) can be put in a compact tensorial form as $\varepsilon^{s}=\mathcal{Q}(\mathbf{P}) \cdot \mathbf{P}$.

Finally, the last part in eq. (1)

$\psi_{\text {elec }}(\mathbf{P}, \mathbf{D})=\frac{1}{2 \kappa_{0}}(\mathbf{D}-\mathbf{P}) \cdot(\mathbf{D}-\mathbf{P})$ 
denotes the electric energy, in which $\kappa_{0}$ is the vacuum permittivity.

\subsection{Equilibrium and constitutive equations}

Let us consider a solid $\Omega$ subjected to surface forces $\mathbf{t}$ on the $\Gamma_{t}$ portion of $\partial \Omega$. In the absence of body forces and assuming quasi static conditions, the mechanical equilibrium is governed by the field equations

$\operatorname{div} \boldsymbol{\sigma}=\mathbf{0} \quad$ in $\Omega, \quad \boldsymbol{\sigma} \cdot \mathbf{n}=\mathbf{t} \quad$ on $\Gamma_{t}$

where $\boldsymbol{\sigma}$ is the Cauchy stress tensor. Additionally, under the assumption of small perturbations, strain is measured with the linear tensor $\varepsilon$ and the displacement field $\mathbf{u}$ is restricted by boundary conditions $\mathbf{u}=\overline{\mathbf{u}}$ on $\Gamma_{u}=\partial \Omega \backslash \Gamma_{t}$. Moreover, having postulated the free energy in the form of (1), stresses and strains are associated by the constitutive equation

$\boldsymbol{\sigma}=\frac{\partial \psi}{\partial \boldsymbol{\varepsilon}}=\frac{\partial \psi_{\text {mech }}}{\partial \boldsymbol{\varepsilon}}=\mathcal{C}:\left(\varepsilon-\varepsilon^{s}\right)$.

A second set of field equations governs the electrical response of the solid $\Omega$ subjected to an imposed electric potential $\bar{\phi}$ on $\Gamma_{\phi} \in \partial \Omega$. The quasi-static form of Maxwell's equations reads

$\operatorname{div} \mathbf{D}=0 \quad$ in $\quad \Omega, \quad \mathbf{D} \cdot \mathbf{n}=-\omega \quad$ on $\quad \Gamma_{\omega}$,

$\mathbf{E}=-\nabla \phi \quad$ in $\Omega, \quad \phi=\bar{\phi} \quad$ on $\quad \Gamma_{\phi}$,

where $\mathbf{E}$ is the electric field vector and $\omega$ is the surface charge density. According to (1), the constitutive equation relating $\mathbf{E}$ and $\mathbf{D}$ is

$\mathbf{E}=\frac{\partial \psi}{\partial \mathbf{D}}=\frac{\partial \psi_{\text {elec }}}{\partial \mathbf{D}}=\frac{1}{\kappa_{0}}(\mathbf{D}-\mathbf{P})$,

which results in the well known formula

$\mathbf{D}=\kappa_{0} \mathbf{E}+\mathbf{P}$.

As in any phase-field method, a crucial point is the formulation of the evolution equation for the order parameter $\mathbf{P}$. A typical intuitive derivation is as follows. Defining the system free energy

$\Psi=\int_{\Omega} \psi \mathrm{d} \Omega$

a relaxation towards the equilibrium condition $\delta \Psi / \delta \mathbf{P}=\mathbf{0}$ is postulated in the form:

$\beta \cdot \frac{\partial \mathbf{P}}{\partial t}=-\frac{\delta \Psi}{\delta \mathbf{P}}$

where $\beta$ is an inverse kinetic tensor related to the domain wall mobility and $\delta \Psi / \delta \mathbf{P}$, defined such that

$\delta \Psi=\frac{\delta \Psi}{\delta \mathbf{P}} \cdot \delta \mathbf{P}+O\|\delta \mathbf{P}\|^{2}$

represents the thermodynamic driving force. Under the assumption that:

$\delta \mathbf{P} \cdot\left(\frac{\partial \psi}{\partial \nabla \mathbf{P}} \cdot \mathbf{n}\right)=0 \quad$ on $\quad \partial \Gamma$ which implies the boundary conditions

$\frac{\partial \psi}{\partial \nabla \mathbf{P}} \cdot \mathbf{n}=\mathbf{0} \quad$ on $\quad \Gamma_{\xi}, \quad \mathbf{P}=\overline{\mathbf{P}} \quad$ on $\quad \Gamma_{P}$,

some algebraic manipulations lead to the well-known time dependent Ginzburg-Landau (TDGL) equation [65]

$\boldsymbol{\beta} \cdot \frac{\partial \mathbf{P}}{\partial t}=\operatorname{div} \frac{\partial \psi}{\partial \nabla \mathbf{P}}-\frac{\partial \psi}{\partial \mathbf{P}}$

It is worth stressing that equation (16), which has been introduced here in the usual heuristic manner, can be justified more rigorously from the point of view of thermodynamics as proposed by Gurtin [66] and $\mathrm{Su}$ and Landis [37]. Their approaches postulate the existence of internal microstresses $\boldsymbol{\xi}$ and internal microforces $\boldsymbol{\pi}$ accounting for the movement of atoms within the lattice and respecting the equilibrium condition

$\operatorname{div} \boldsymbol{\xi}+\boldsymbol{\pi}=\mathbf{0}$ in $\Omega$.

An application of the second law of thermodynamics shows that microstresses are work-conjugate of $\nabla \mathbf{P}$, and that microforces can be divided in two contributions

$\boldsymbol{\xi}=\frac{\partial \psi}{\partial \nabla \mathbf{P}}, \quad \boldsymbol{\pi}=\boldsymbol{\eta}-\boldsymbol{\beta} \cdot \frac{\partial \mathbf{P}}{\partial t}, \quad \boldsymbol{\eta}=\frac{\partial \psi}{\partial \mathbf{P}}$.

Substituting equations (18) into the microforce equilibrium (17), one obtains again equation (16).

Remark. In the present model, the total polarization is used as "order parameter". It is worth stressing that there are other possible choices [67]. For instance, in the works of $\mathrm{Li}$ et al. [54, 55, 56, 57, 59] and Wang et al. $[34,52,50,51]$, the spontaneous polarization is regarded as the order parameter. In such kind of phase-field models, the spontaneous polarization is embedded in a background material, for instance in the paraelectric phase material. For simplicity, the total polarization $\mathbf{P}$ is divided into two components: the spontaneous polarization $\mathbf{P}^{s}$ and the induced polarization $\mathbf{P}^{i}$, assumed linearly proportional to the electric field [52]

$\mathbf{P}^{i}=\kappa_{0} \chi_{e} \mathbf{E}=\kappa_{0}\left(\kappa_{r}-1\right) \mathbf{E}$.

where $\chi_{e}=\kappa_{r}-1$ is the electric susceptibility and $\kappa_{r}$ is the relative permittivity. Consequently, the dielectric displacement becomes

$\mathbf{D}=\kappa_{0} \mathbf{E}+\left(\mathbf{P}^{i}+\mathbf{P}^{s}\right)=\kappa_{0} \kappa_{r} \mathbf{E}+\mathbf{P}^{s}$

\section{Finite Elements implementation}

The multi-field problem is formulated in terms of the unknown fields $\mathbf{u}, \phi$ and $\mathbf{P}$, which are subjected to the boundary conditions discussed in the previous section $\mathbf{u}=\overline{\mathbf{u}} \quad$ on $\Gamma_{u}, \quad \phi=\bar{\phi} \quad$ on $\Gamma_{\phi}, \quad \mathbf{P}=\overline{\mathbf{P}} \quad$ on $\Gamma_{P}$. 
Their functional spaces, endowed with sufficient continuity and respecting the boundary conditions in strong form, are denoted $\mathcal{C}_{u}(\overline{\mathbf{u}}), \mathcal{C}_{\phi}(\bar{\phi})$ and $\mathcal{C}_{P}(\overline{\mathbf{P}})$, respectively. While constitutive equations are again enforced in a strong form, equilibrium conditions are imposed in a weak manner using as test functions

$$
\tilde{\mathbf{u}} \in \mathcal{C}_{u}(\mathbf{0}), \quad \tilde{\phi} \in \mathcal{C}_{\phi}(0), \quad \tilde{\mathbf{P}} \in \mathcal{C}_{P}(\mathbf{0}),
$$

where the spaces $\mathcal{C}_{u}(\mathbf{0}), \mathcal{C}_{\phi}(0)$ and $\mathcal{C}_{P}(\mathbf{0})$, collect functions vanishing on $\Gamma_{u}, \Gamma_{\phi}$ and $\Gamma_{P}$, respectively. Multiplying equations (7), (9) and (17) by the respective test functions, integrating over $\Omega$ and enforcing the boundary equilibrium conditions

$$
\begin{aligned}
& \boldsymbol{\sigma} \cdot \mathbf{n}=\mathbf{t} \quad \text { on } \quad \Gamma_{t}, \quad \mathbf{D} \cdot \mathbf{n}=-\omega \quad \text { on } \quad \Gamma_{\omega}, \\
& \boldsymbol{\xi} \cdot \mathbf{n}=\mathbf{0} \quad \text { on } \Gamma_{\xi},
\end{aligned}
$$

some standard algebraic manipulations lead to the final weak form of the equilibrium conditions

find $\mathbf{u}(\mathbf{x}) \in \mathcal{C}_{u}(\overline{\mathbf{u}})$ such that, $\forall \tilde{\mathbf{u}} \in \mathcal{C}_{u}(\mathbf{0})$

$\int_{\Omega} \boldsymbol{\sigma}: \tilde{\varepsilon} \mathrm{d} \Omega-\int_{\Gamma_{t}} \mathbf{t} \cdot \tilde{\mathbf{u}} \mathrm{d} \Gamma=0$

$$
\text { find } \phi(\mathbf{x}) \in \mathcal{C}_{\phi}(\bar{\phi}) \text { such that, } \forall \tilde{\phi} \in \mathcal{C}_{\phi}(0)
$$

$\int_{\Omega} \mathbf{D} \cdot \tilde{\mathbf{E}} \mathrm{d} \Omega+\int_{\Gamma_{\omega}} \omega \tilde{\phi} \mathrm{d} \Gamma=0$

find $\mathbf{P}(\mathbf{x}, t) \in \mathcal{C}_{P}(\overline{\mathbf{P}})$ such that, $\forall \tilde{\mathbf{P}} \in \mathcal{C}_{P}(\mathbf{0})$

$$
\begin{aligned}
\int_{\Omega}\left[\left(\boldsymbol{\beta} \cdot \frac{\partial \mathbf{P}}{\partial t}\right) \cdot \tilde{\mathbf{P}}+\boldsymbol{\xi}: \nabla \tilde{\mathbf{P}}+\boldsymbol{\eta} \cdot \tilde{\mathbf{P}}\right] & \mathrm{d} \Omega \\
\forall t & =0 \\
\forall t & \in[0, T] .
\end{aligned}
$$

Adopting Voigt's notation, we write the constitutive laws as

$$
\begin{aligned}
\{\boldsymbol{\sigma}\}= & {[\mathbf{C}](\{\boldsymbol{\varepsilon}\}-[\mathbf{Q}(\mathbf{P})]\{\mathbf{P}\}), } \\
\{\mathbf{D}\}= & {[\boldsymbol{\kappa}]\{\mathbf{E}\}+\{\mathbf{P}\}, } \\
\{\boldsymbol{\xi}\}=[\mathbf{G}]\{\nabla \mathbf{P}\}, & \\
\{\boldsymbol{\eta}\}= & \left([\boldsymbol{\alpha}(\mathbf{P})]+2[\mathbf{Q}(\mathbf{P})]^{\mathrm{T}}[\mathbf{C}][\mathbf{Q}(\mathbf{P})]\right)\{\mathbf{P}\} \\
& -2[\mathbf{Q}(\mathbf{P})]^{\mathrm{T}}[\mathbf{C}]\{\boldsymbol{\varepsilon}\}-\{\mathbf{E}\} .
\end{aligned}
$$

A detailed description of all the terms in equations (24)-(27) is presented in Appendix B for 2D problems. Equations (21)-(23) can be now expressed in matrix form as

$$
\begin{aligned}
& \int_{\Omega}\left(\{\tilde{\boldsymbol{\varepsilon}}\}^{\mathrm{T}}[\mathbf{C}]\{\boldsymbol{\varepsilon}\}-\{\tilde{\boldsymbol{\varepsilon}}\}^{\mathrm{T}}[\mathbf{C}][\mathbf{Q}(\mathbf{P})]\{\mathbf{P}\}\right) \mathrm{d} \Omega= \\
& \int_{\Gamma_{t}}\{\tilde{\mathbf{u}}\}^{\mathrm{T}}\{\mathbf{T}\} \mathrm{d} \Gamma \\
& \int_{\Omega}\left(\{\tilde{\mathbf{E}}\}^{\mathrm{T}}[\boldsymbol{\kappa}]\{\mathbf{E}\}+\{\tilde{\mathbf{E}}\}^{\mathrm{T}}\{\mathbf{P}\}\right) \mathrm{d} \Omega=-\int_{\Gamma_{\omega}} \tilde{\phi} \omega \mathrm{d} \Gamma, \\
& \int_{\Omega}\left[\{\tilde{\mathbf{P}}\}^{\mathrm{T}}[\boldsymbol{\beta}]\{\dot{\mathbf{P}}\}+\{\nabla \tilde{\mathbf{P}}\}^{\mathrm{T}}[\mathbf{G}]\{\nabla \mathbf{P}\}\right. \\
& +\{\tilde{\mathbf{P}}\}^{\mathrm{T}}\left([\boldsymbol{\alpha}(\mathbf{P})]+2[\mathbf{Q}(\mathbf{P})]^{\mathrm{T}}[\mathbf{C}][\mathbf{Q}(\mathbf{P})]\right)\{\mathbf{P}\} \\
& \left.-2\{\tilde{\mathbf{P}}\}^{\mathrm{T}}[\mathbf{Q}(\mathbf{P})]^{\mathrm{T}}[\mathbf{C}]\{\boldsymbol{\varepsilon}\}-\{\tilde{\mathbf{P}}\}^{\mathrm{T}}\{\mathbf{E}\}\right] \mathrm{d} \Omega=0,
\end{aligned}
$$

where $\dot{\mathbf{P}}=\partial \mathbf{P} / \partial t$.

\subsection{Space discretization}

We restrict ourselves to $2 \mathrm{D}$ problems in which the polarization and the electric field have only in-plane components. The space $\Omega$ is discretized with six node triangles. Every node is associated to five degrees of freedom: two displacement components, one electrical potential and two polarization components. In each element the fields are interpolated using quadratic shape functions for the displacements and the electrical potential, whereas we select a linear interpolation for the polarization. This choice is due to the fact that the polarization is strictly connected to the strain and to the electric fields, which are the gradients of the displacements and potential, respectively.

Collecting all the unknowns in the arrays $\{\mathbb{U}\},\{\Phi\},\{\mathbb{P}\}$, the discretized weak form generates a system of non-linear equations:

$$
\begin{aligned}
& {\left[\mathbb{K}_{\mathrm{U}}\right]\{\mathbb{U}\}+\left[\mathbb{K}_{\mathrm{UP}}(\mathbb{P})\right]\{\mathbb{P}\}=\left\{\mathbb{F}_{\mathrm{U}}\right\} ;} \\
& {\left[\mathbb{K}_{\Phi}\right]\{\Phi\}+\left[\mathbb{K}_{\Phi \mathrm{P}}(\mathbb{P})\right]\{\mathbb{P}\}=\left\{\mathbb{F}_{\Phi}\right\} ;} \\
& {[\mathbb{M}]\{\dot{\mathbb{P}}\}+\left(\left[\mathbb{K}_{\mathrm{G}}\right]+\left[\mathbb{K}_{\mathrm{P}}(\mathbb{P})\right]\right)\{\mathbb{P}\}} \\
& \quad+\left[\mathbb{K}_{\mathrm{PU}}(\mathbb{P})\right]\{\mathbb{U}\}+\left[\mathbb{K}_{\mathrm{P} \Phi}\right]\{\Phi\}=\{0\} .
\end{aligned}
$$

\subsection{Time discretization}

We adopt a simple staggered approach for the time marching integration. Assuming that the state at $t_{n}$ is known, equations (31)-(32) are imposed at time $t_{n+1}$ with the following explicit scheme:

$$
\begin{aligned}
& {\left[\mathbb{K}_{\mathrm{U}}\right]\left\{\mathbb{U}_{n+1}\right\}=\left\{\mathbb{F}_{\mathrm{U}}\right\}-\left[\mathbb{K}_{\mathrm{UP}}\left(\mathbb{P}_{n}\right)\right]\left\{\mathbb{P}_{n}\right\} ;} \\
& {\left[\mathbb{K}_{\phi}\right]\left\{\Phi_{n+1}\right\}=\left\{\mathbb{F}_{\phi}\right\}-\left[\mathbb{K}_{\Phi \mathrm{P}}\left(\mathbb{P}_{n}\right)\right]\left\{\mathbb{P}_{n}\right\} .}
\end{aligned}
$$

The time derivative of the polarization vector is approximated by finite differences:

$$
\{\dot{\mathbb{P}}\}=\frac{\left\{\mathbb{P}_{n+1}-\mathbb{P}_{n}\right\}}{\Delta t} \text {. }
$$

With a forward Euler method, equation (33) becomes:

$$
\begin{gathered}
{[\mathbb{M}]\left\{\mathbb{P}_{n+1}\right\}=\left([\mathbb{M}]-\Delta t\left(\left[\mathbb{K}_{\mathrm{G}}\right]+\left[\mathbb{K}_{\mathrm{P}}\left(\mathbb{P}_{n}\right)\right]\right)\right)\left\{\mathbb{P}_{n}\right\}} \\
-\Delta t\left(\left[\mathbb{K}_{\mathrm{PU}}\left(\mathbb{P}_{n}\right)\right]\left\{\mathbb{U}_{n+1}\right\}+\left[\mathbb{K}_{\mathrm{P} \Phi}\right]\left\{\Phi_{n+1}\right\}\right),
\end{gathered}
$$

in which $\left\{\mathbb{U}_{n+1}\right\}$ and $\left\{\Phi_{n+1}\right\}$ are the solution of equations (34)-(35).

\section{Simulation results}

The long term motivation of this investigation is the simulation of thin films deposited on bulk materials for MEMS applications. Trying to limit the computational burden as much as possible, we consider a 2D section of a thin layer of piezo-material extending indefinitely in the out-of-plane direction and in the $x_{1}$ direction. 


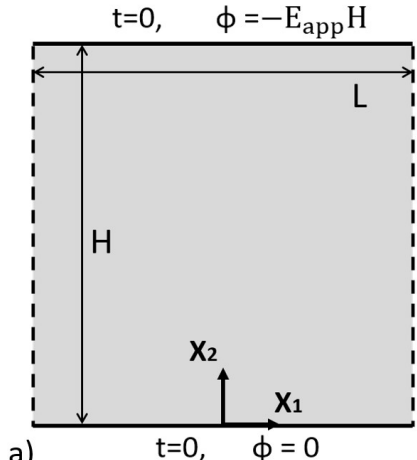

a)

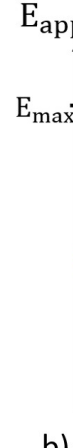

b)

Figure 2: Simulation model: a) Boundary conditions; b) Applied electric field

According to [37] we assume a generalized plane strain condition:

$P_{3}=0, \quad E_{3}=0, \quad \varepsilon_{13}=\varepsilon_{23}=0, \varepsilon_{33}=\varepsilon_{\perp}$

where $\varepsilon_{\perp}$ is the normal spontaneous strain, i.e. the remnant $\varepsilon_{33}$ strain due to an in-plane spontaneous polarization when the ferroelectric material transforms from the cubic phase to the tetragonal phase. E.g., for the material considered in the present investigation, (see Appendix A), $\varepsilon_{\perp}=-0.0149$. With this condition we guarantee zero out-of-plane stress in the initial configuration where we assume absence of external mechanical and electrical loading; on the contrary any further contribution to the $\varepsilon_{33}$ strain is physically prevented by the "infinite" extension of the film in the $x_{3}$ direction.

Since in this investigation we do not consider the presence of the substrate, the bottom and top surfaces are treated as traction free boundaries. On the contrary, in order to simulate a sufficiently long extension of the thin film in the $x_{1}$ direction, a rectangular strip of PTO material of size $L \times H$ is modelled and suitable periodic boundary conditions are enforced on the vertical sides:

$u_{1}\left(L / 2, x_{2}\right)=u_{1}\left(-L / 2, x_{2}\right)+L<\varepsilon_{11}>$,

$u_{2}\left(L / 2, x_{2}\right)=u_{2}\left(-L / 2, x_{2}\right)$,

$\phi\left(L / 2, x_{2}\right)=\phi\left(-L / 2, x_{2}\right)$,

$P_{1}\left(L / 2, x_{2}\right)=P_{1}\left(-L / 2, x_{2}\right)$,

$P_{2}\left(L / 2, x_{2}\right)=P_{2}\left(-L / 2, x_{2}\right)$,

where $\left\langle\varepsilon_{11}\right\rangle$ is an average stretch along the $x_{1}$ direction which plays a key role in the analyses. In the presence of a stiff substrate the value of $\left\langle\varepsilon_{11}\right\rangle$ would be imposed to match that of the substrate itself, since it is known $([53,68])$ that this has an important impact on the hystereris loop. However, in the present context we rather focus on the effect of defects and $\left\langle\varepsilon_{11}\right\rangle$ is defined in order to guarantee a sort of "stress free" condition at the vertical borders. The issue of inserting stress free conditions in periodic bcs has been clarified in [69] where a generic 3D periodic cell is addressed. Here, limiting ourselves to 2D problems, we adopt a choice which appears as a modification of their ASSF (Adaptive Spherical Stress Free) option and consists in setting (see eq.(5)):

$$
<\varepsilon_{11}>=<\varepsilon_{11}^{s}>=Q_{11}<P_{1}^{2}>+Q_{12}<P_{2}^{2}>
$$

Basically we aim at letting the material expand freely in the $x_{1}$ direction when the spontaneous "inelastic" strain $\varepsilon_{11}^{s}$ appear. The value of $\left\langle\varepsilon_{11}\right\rangle$ clearly evolves during the analysis. One may argue that also $u_{2}$ should be modified consistently in order to account for $\left\langle\varepsilon_{12}^{s}\right\rangle$. However, its physical meaning is less evident and, as done in [69], we prefer to disregard the contribution of off-diagonal components of $\varepsilon^{s}$.

Rigid body movements of the specimen are isostatically prevented. The electrical potential is set to zero at the bottom and $\phi=-E_{\text {app }} H$ at the top, where $E_{\text {app }}$ is the applied electric field in the vertical direction, simulating the presence of the electrodes. All the analyses addressed in this Section start with a preliminary phase where the specimen is initially completely polarized in the $x_{1}$ direction and relaxes towards an equilibrium configuration with $E_{\text {app }}=0$. It is worth stressing that this state could differ from the trivial solution in the presence of defects. In subsequent phases, the electric field is applied in the vertical direction and its value is increased or decreased step by step by $\Delta E$. During each loading step the electric field is kept constant (see figure 2(b)) until the polarization field relaxes to a steady state. The first electrical loading steps tend to polarize the material in the $x_{2}$ direction (poling). Then, the classical hysteresis loop $P$ vsE is simulated. It is worth stressing that the initial condition $P_{1}=P^{s}$ and $P_{2}=0$ has been chosen only for simplicity. Other options, like random polarization with magnitude between 0 and $P^{s}$, have been tested. These lead to different initial relaxed configurations, but eventually the same macroscopic hysteresis loop is obtained. 
The material coefficients utilized in the simulations are listed in Appendix A. The selected value of the gradient coefficient $G_{11}$ is such that the domain wall thickness is approximatively $1.5 \mathrm{~nm}$. Assuming that three quadratic triangular elements are sufficient to capture the sharp interface, the typical finite element mesh size is set to $0.5 \mathrm{~nm}$ in all the analyses performed. With this refinement level, no numerical artefacts like mesh pinning have been observed.

\subsection{Perfect single crystal}

As a first example, figure 3 presents the hysteresis loop computed for a single square crystal of $L=H=$ $20 \mathrm{~nm}$ made of PTO without any imperfection. In this example, the whole domain simultaneously switches during the electrical loading and the polarization is homogeneous.

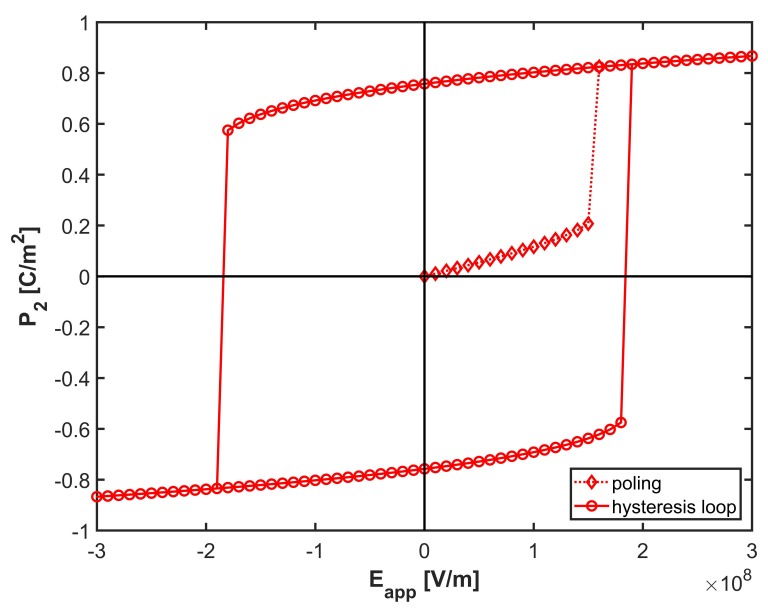

Figure 3: Hysteresis loop for a perfect single crystal.

For a perfect single crystal the value of the remnant polarization $P_{r}$ is equal to $0.757 \mathrm{C} / \mathrm{m}^{2}$, i.e. it coincides the value of the spontaneous polarization $P^{s}$. The coercive switching fields are $E_{C 90}=1.52 \times 10^{8} \mathrm{~V} / \mathrm{m}$ and $E_{C 180}=1.89 \times 10^{8} \mathrm{~V} / \mathrm{m}$, for $90^{\circ}$ and $180^{\circ}$ switching processes, respectively.

The computed coercive field strength $E_{C 180}$ is about two orders of magnitude higher than the experimentally measured coercive fields of real ferroelectric ceramics [17, 70]. Indeed, the simulated problem represents an idealized system, whereas real ferroelectric materials contain numerous imperfections and are typically polycrystalline with a complicated grain structure. These mechanisms have a strong impact on the coercive field strength [18], as investigated in the subsequent sections.

\subsection{Defects in a single crystal}

The aim of this section is to study the evolution of ferroelectric domains and their interaction with some typical defects or imperfections in the materials. In particular, the effects on the global $P \mathrm{vs} E$ hysteresis loop are investigated. Since it is extremely difficult to interpret the experimental observations when all defects are considered simultaneously, in the following we will focus sequentially on a single crystal of size $L=H=20 \mathrm{~nm}$ with three different types of defects: voids of different shape inside the sample; charged point defects, reflecting for instance the presence of an oxygen vacancy; pinning of the polarization.

4.2.1. Voids in the material. Let us consider the examples in figure 4 where a square hole (case a), a circular hole (case b) and four holes (case c), have been inserted in the perfect crystal. We set $d=5 \mathrm{~nm}$ in case (a) and $R=2.82 \mathrm{~nm}$ in case (b), whereas in the last case $d$ and $R$ are halved, so as to maintain the total area of the voids $A=25 \mathrm{~nm}^{2}$ invariant in the three situations.

The electrical conditions on the hole boundaries are open-circuited, i.e. $\mathbf{D} \cdot \mathbf{n}=0$, where $\mathbf{n}$ is the outward normal. This is a reasonable approximation when the medium in the void has a low permittivity (e.g. air). Figure 5 shows the hysteresis loop and the domain evolution for case (a). Even with zero applied electric field, starting from the initial configuration completely polarized in the horizontal direction, $P_{1}=$ $P^{s}$ and $P_{2}=0$, the polarization relaxes towards a multi-domain configuration (1). In particular, two domains with vertical but opposite polarization are nucleated between the hole and the corners. This is induced by the orientation of the surfaces of the square void which coincide with the possible orientations of spontaneous polarization. Therefore, $\mathbf{P}$ tends to orient itself parallel to these surfaces thus satisfying the boundary condition $\mathbf{D} \cdot \mathbf{n}=0$ in an ideal way. Then, when a positive vertical electric field is applied, the polarization vector switches almost totally in the vertical positive direction (2). It is possible to observe that small regions of opposite polarization persist near the void borders. It is worth remarking that even when $E_{\text {app }}=0$ in the hysteresis loop, the presence of the void induces multi-domain configurations (3),(5). In particular, many head to head and tail to tail $90^{\circ}$ domain walls appear. This is usually considered to be very unlikely from the physical point of view since surface charges of equal sign face each other. However, these domain configurations are enforced by the periodic boundary conditions. Furthermore, this evolution of the polarization is qualitatively in accordance with the results presented in literature $[61,71]$. 


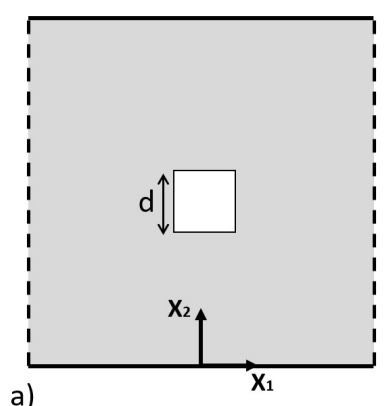

a)

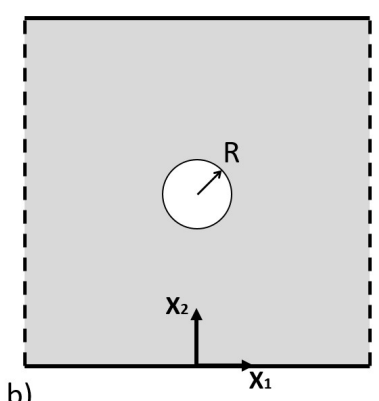

b)

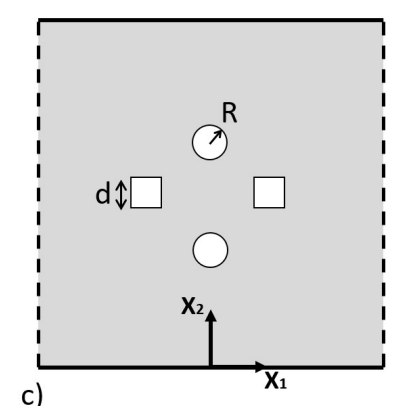

c)

Figure 4: Samples with a) a square hole, b) a circle hole and c) four holes.

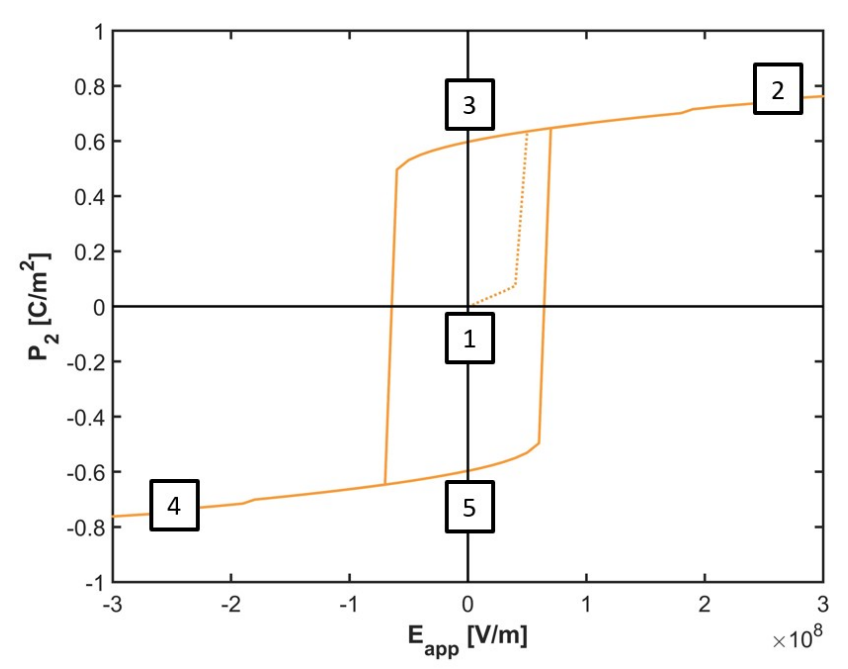

(a) Hysteresis loop PvsE
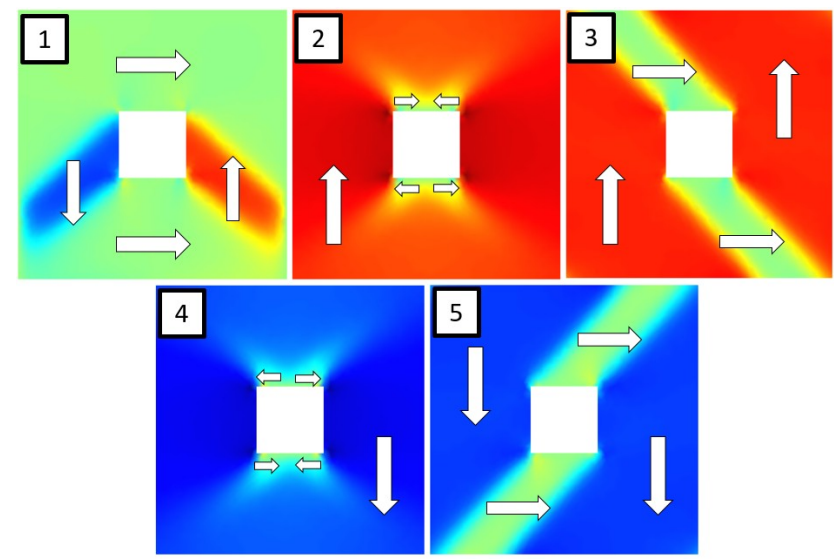

(b) Domain evolution

Figure 5: Specimen with a square hole (case a): a) hysteresis loop and b) contour plots for $P_{2}$. The arrows in the domain evolution indicate the orientation of polarization vector.

Next we consider case (b) with a circular hole having the same boundary conditions. It can be appreciated from figure 6 that the shape of hysteresis loop is quite different with respect to the previous case.

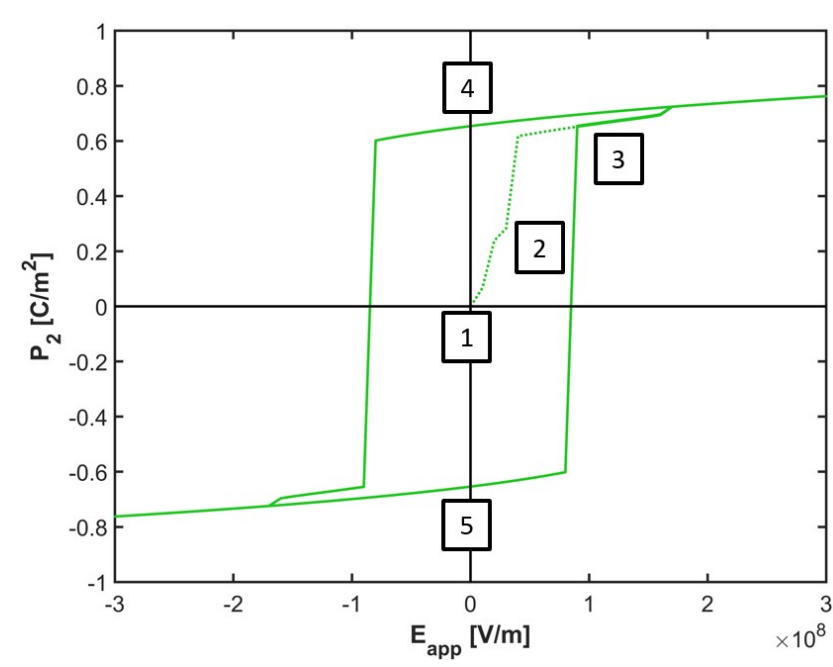

(a) Hysteresis loop PvsE
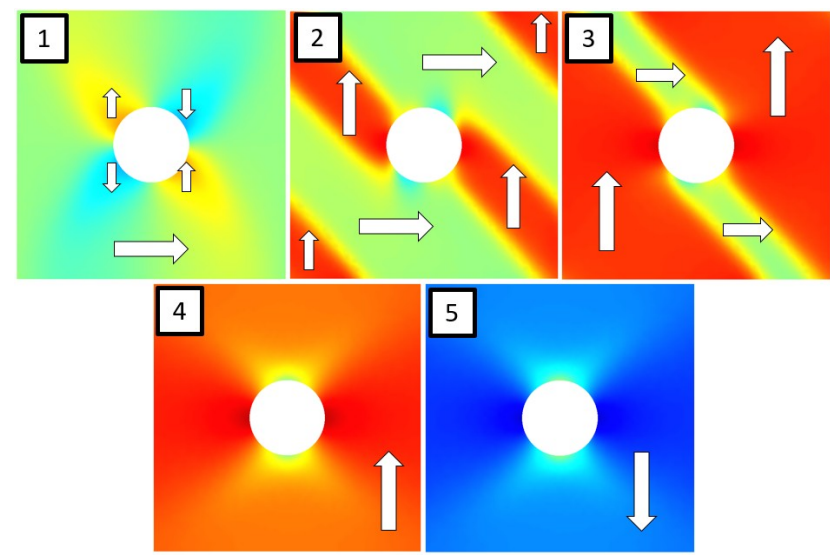

(b) Domain evolution

Figure 6: Specimen with a circular hole (case b): a) hysteresis loop and b) contour plots for $P_{2}$. The arrows in the domain evolution indicate the orientation of polarization vector.

Now the void-surface normal is no longer aligned with the directions of spontaneous polarization. Initially, four small vertical domains appear in a symmetric way (1), resulting in a zero macroscopic vertical 


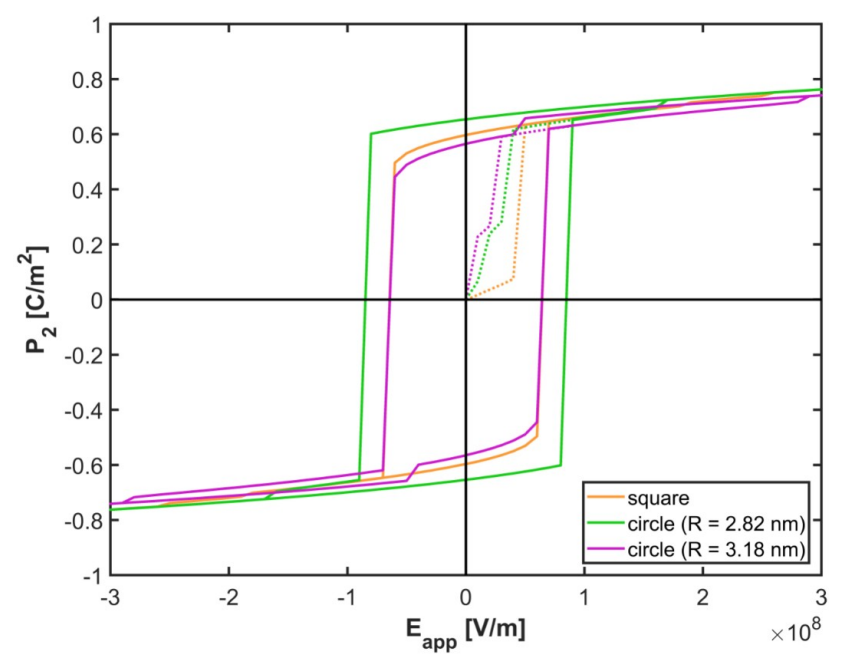

Figure 7: Comparison between the hysteresis loops for two different values of $R$ in the circle case (b) and the square case (a).

polarization. Then, the domains get progressively aligned with the imposed electric field (2),(3) until they switch almost completely. In the hysteresis loop, when $E_{\text {app }}=0$ the polarization is almost homogeneous and aligned in the direction of the applied electric field $(4),(5)$, with the exception of small regions near the circular hole.

However, the microstructure evolution in figures $5 \mathrm{~b}$ and $6 \mathrm{~b}$ reveals that domains nucleate around the defect core and their extension and importance could depend on the perimeter of the void rather than its area. Still considering a single circular defect, we therefore set $R=3.18 \mathrm{~nm}$ in order to force the same perimeter $(2 p=20 \mathrm{~nm})$ as for the square defect. From figure 7 we can see how the values of the remnant polarization $P_{r}$ and the coercive field $E_{C 180}$ for the "equal perimeter circle" are lower than for the "equal area circle" and globally more similar to the square case (a).

Case (c) generates an hysteresis loop (see figure 8) that is a sort of average of the two previous examples: it has the same shape as case (b), but the same value of coercive field $E_{C 180}$ as case (a). The domain evolution is clearly affected by the interaction between the different holes in the material.

Finally, figure 9 shows the hysteresis loops for the three examples in figure 4 with the total area of the voids as invariant, compared with the perfect single crystal case.

Despite its very qualitative nature, these examples highlight the potential effects of voids in the material. Even if the void area represents only $6.35 \%$ of the total area of the specimen analysed, the reduction of the strength of the coercive field exceeds $50 \%$ in cases (a)

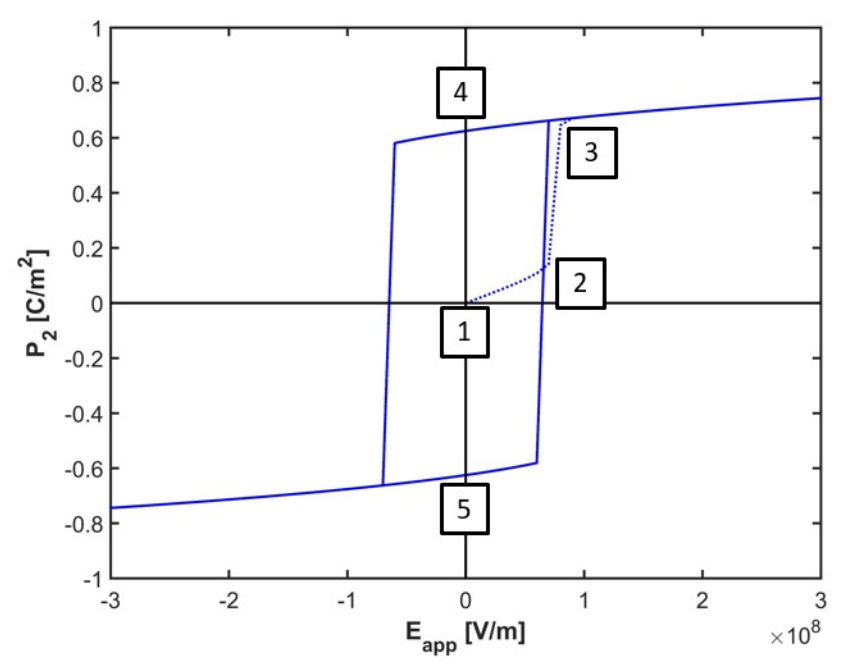

(a) Hysteresis loop PvsE

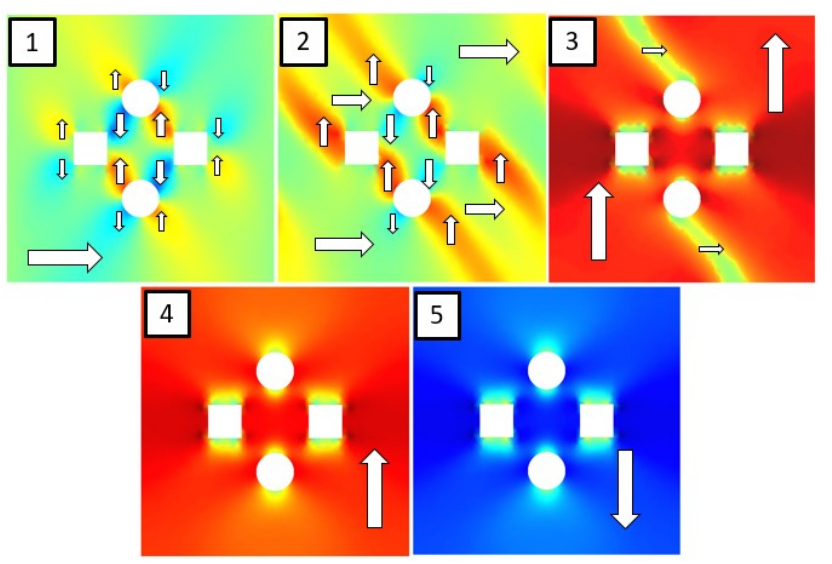

(b) Domain evolution

Figure 8: Specimen with four holes (case c): a) hysteresis loop and b) contour plots for $P_{2}$. The arrows in the domain evolution indicate the orientation of polarization vector.

and (c), less in case (b).

4.2.2. Charged point defect. Let us consider now a sample containing a defect of charge $q$ placed in its center. Typically, a point charge positioned at $\overline{\mathbf{x}}$ is described through the Dirac delta function

$\rho(\mathbf{x})=q \delta(\mathbf{x}-\overline{\mathbf{x}})$

where $\rho$ is the volume density of the charge, which is included into the Maxwell's equation (9) as $\operatorname{div} \mathbf{D}-\rho=$ 0 . In a two-dimensional model, the electric point charge is a line charge extending to infinity in the $x_{3}$ direction. Setting $q=5 \times 10^{-9} \mathrm{C} / \mathrm{m}$ and proceeding as described before, we obtain the hysteresis loop $P$ vs $E$ and the domain patterns depicted in figure 10 .

The presence of the charge reduces both the strength of the coercive field and the value of the 


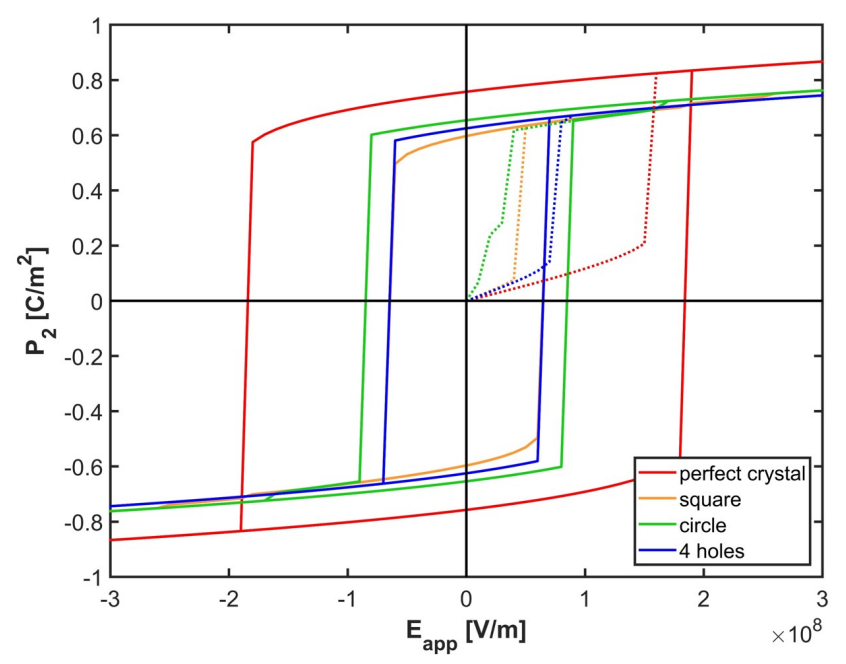

Figure 9: Comparison between hysteresis loops for the three samples in Figure 4 and the perfect crystal.

remnant polarization compared to the case of the perfect crystal. In a sense, the presence of the charge promotes and facilitate the nucleation of ferroelectric domains at the site of the charged defect, as described in the following. Initially, even for $E_{\text {app }}=0$, a domain in the negative vertical direction is generated between the charge and one corner (1). When the electric field increases, a new domain (2) gets rapidly oriented like the applied electric field and it starts the $90^{\circ}$ switching process. Next the polarization progresses towards a homogeneous state for high values of $E_{\text {app }}(3)$, but for a small area in the vicinity of the charge. When the electric field decreases to zero, switching is triggered by the fast reorientation in the $x_{1}$ direction (4) of a domain pinned at the point charge. The process then progresses symmetrically for negative $E_{\text {app }}$.

As a validation of the present implementation of the PFM, we compare our finite element simulations with results presented by Völker et al. in [62], in which the numerical analyses have been performed in COMSOLMultiphysics (C). Here, the material parameters have been chosen following [46, 62] for PZT material. Figure 11 shows the comparison of the coercive field strengths computed in the two implementations, showing an excellent agreement. It is worth stressing that for an increasing charge density, both coercive field strengths, $E_{C 90}$ and $E_{C 180}$, are reduced significantly. In particular, the coercive field $E_{C 180}$ is affected more by the defect than $E_{C 90}$ and the two values converge for high charge values. This could be possibly explained by noting that the concentrated charge actually separates the $180^{\circ}$ switching process in two $90^{\circ}$ sub-processes.

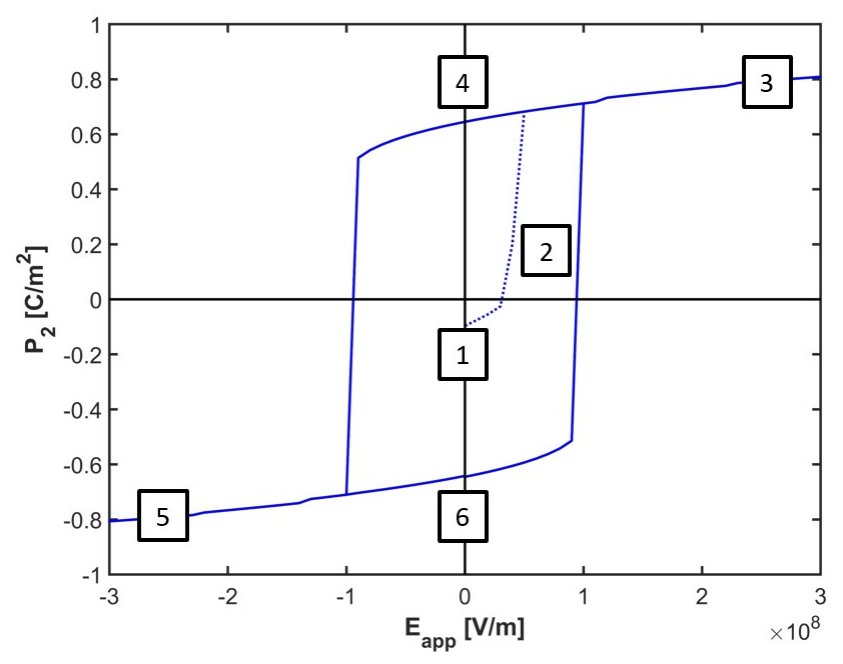

(a) Hysteresis loop PvsE

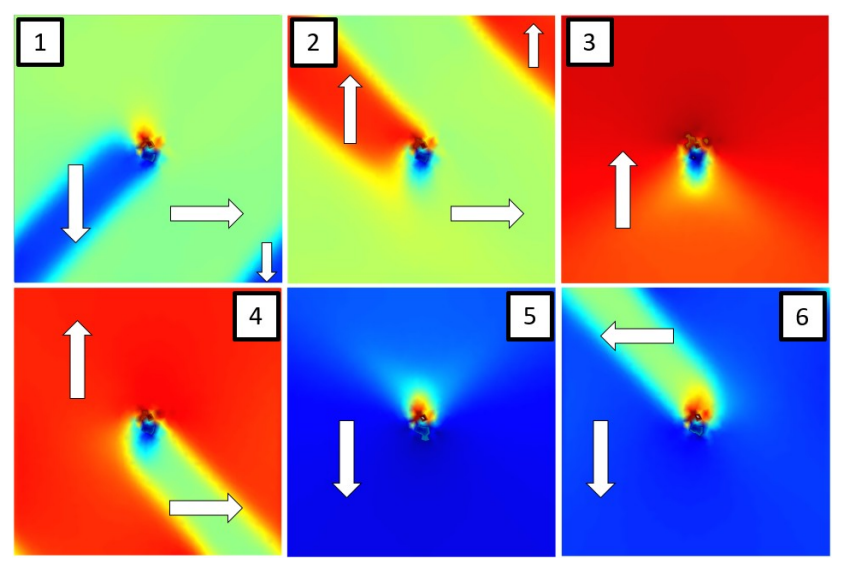

(b) Domain evolution

Figure 10: Specimen with a charge $q=5 \times 10^{-9} \mathrm{C} / \mathrm{m}$ in its center: a) hysteresis loop and b) contour plots for $P_{2}$. The arrows in the domain evolution indicate the orientation of polarization vector.

4.2.3. Polarization pinning. Ferroelectric materials are subjected to degradation in time even in the absence of external loading. This phenomenon is commonly defined as aging. Typically, the most common reason for aging is the migration of charged defects, like oxygen vacancies, which stabilize a given domain configuration, hindering the polarization reversal when an electric field is applied [22]. The simplest way to reproduce this situation is to consider a polarization defect in the form of a region where the polarization is pinned in one configuration without any possibility to evolve. Therefore, we simulate a square sample of area $A=25 \mathrm{~nm}^{2}$, in which the polarization is oriented in $x_{1}$ direction $\left(\mathbf{P}=\left(P^{s} ; 0\right)\right)$, that is orthogonal to the applied electric field (figure 12).

The hysteresis loop and the domain patterns obtained in the presence of this polarization defect is 


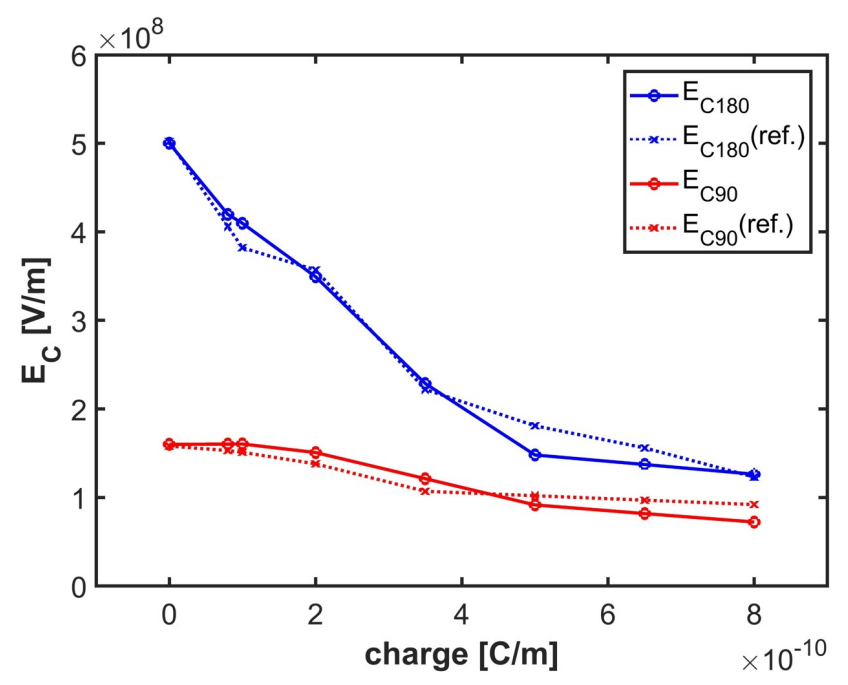

Figure 11: Comparison of the values of the coercive fields computed for several charge densities with the present model (straight line) vs. those presented in [62] (dashed line). PZT material.

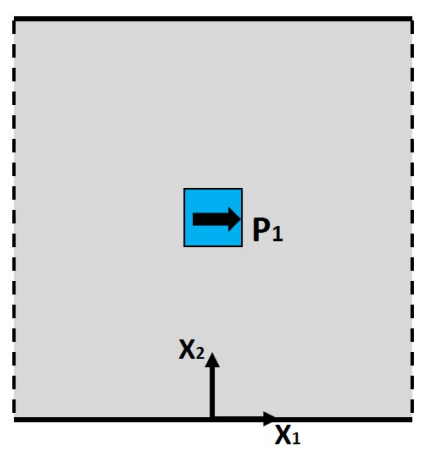

Figure 12: Sample with a region of area $A=25 \mathrm{~nm}^{2}$ where the polarization is pinned in $x_{1}$ direction.

shown in figure 13. Initially the polarization is oriented completely in the horizontal direction (1), as in the case of a perfect single crystal. Applying a positive electric field, multidomain configurations (2),(3) are generated, but when the electric field is removed the specimen recovers the initial condition with uniform polarization in $x_{1}$ direction, resulting in an unpoled state in the hysteresis loop PvsE. This happens because continuity at the boundaries of the pinned area drives the system back into a state of minimum energy with $P_{2}=0$. For a negative electric field, we observe a similar behaviour of the hysteresis loop (4),(5), which eventually features two totally distinct and symmetric cycles.

It is worth stressing note that the shape of the obtained loop is very similar to the response of an aged ferroelectric (see figure 1a), thus strongly supporting the initial guess that aging might be also associated to polarization pinning.

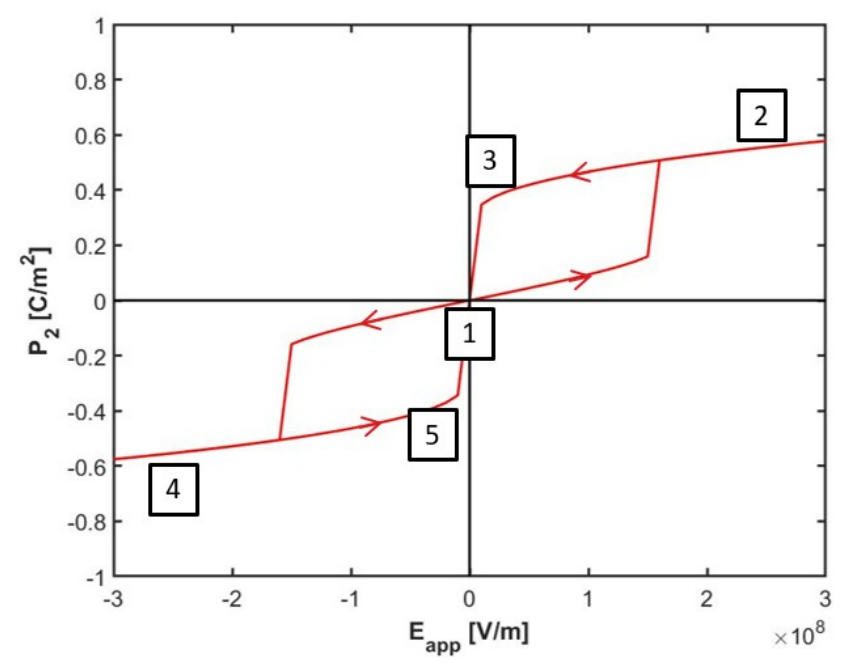

(a) Hysteresis loop PvsE

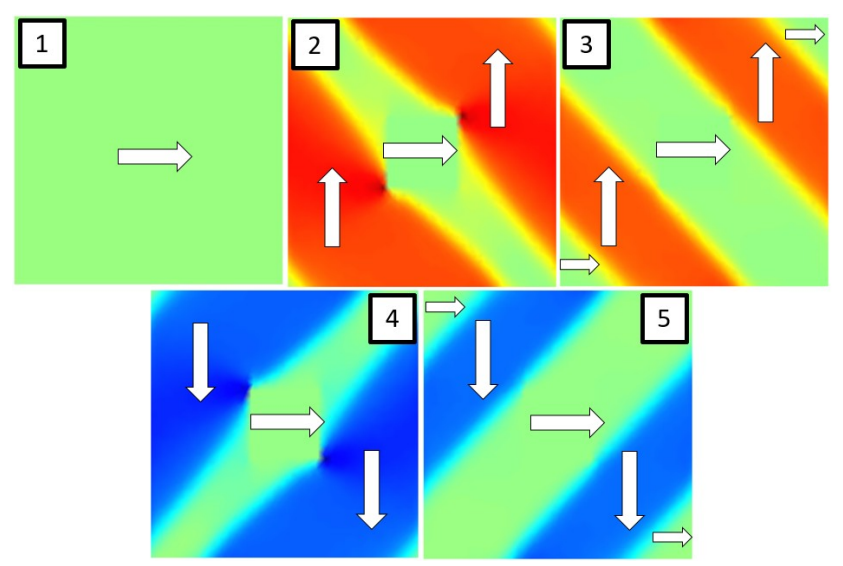

(b) Domain evolution

Figure 13: Sample with a pinning area in its center: a) hysteresis loop and b) contour plots for $P_{2}$. The arrows in the domain evolution indicate the orientation of polarization vector.

Another approach to simulate a pinned polarized domain would be to consider an electric dipole in the sample by placing two charge defects with opposite sign separated by a distance $\mathbf{d}$, inducing an electric dipole moment $\mathbf{p}=q \mathbf{d}=\mathbf{P} A$. In order to compare the two approaches, we consider two charges with $q=3.75 \times 10^{-9} \mathrm{C} / \mathrm{m}$ placed in $\mathrm{x}=(-2.5 ; 5) \mathrm{nm}$ and $\mathbf{x}=(2.5 ; 5) \mathrm{nm}$, respectively. The resulting hysteresis loop is shown in figure 14 indicating a qualitative accordance with the first simplified approach, thus supporting our conclusions. However, the presence of an electric dipole does not prevent the polarization to evolve in the vicinity of the dipole itself when the electric field changes direction. Therefore, we consider the first approach, although simplistic, more appropriate to simulate the "aging" condition. 


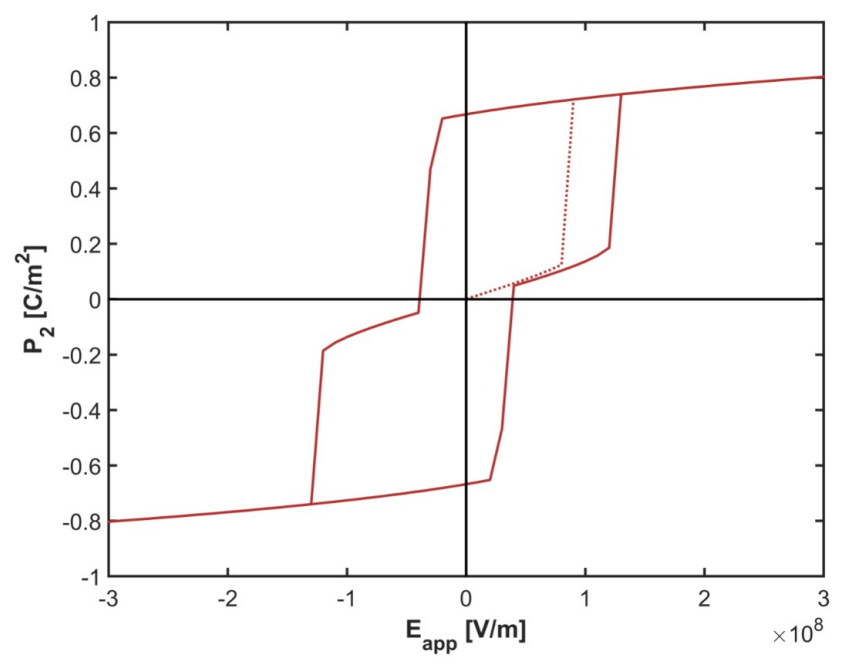

Figure 14: Hysteresis loop for a sample with an electric dipole.

\subsection{Polycrystalline material}

Most ferroelectrics are polycrystals composed of several grains having different orientations and separated by grain boundaries. The orientation of the grains affects the elastic and electrostatic interactions, whereas the presence of grain boundaries generates depolarizing fields. Therefore, the multigrain structure plays an important role in determining the domain configuration inside the material. We aim at showing how the multigrain nature of ferroelectric polycrystalline can impact on their properties, and in primis on the hysteresis loop.

In the FEM modelling discussed in Section 3 the polarization vector, as well as the displacements and the potential, is continuous across elements, following $[50,51]$. This common choice has some important consequences. When the interface between grains has "zero" thickness (section 4.3.1), $\mathbf{P}$ is continuous across grains, which might be criticized from a physical point of view. On the contrary, in section 4.3 .2 we simulate interfaces between grains with finite thickness and consider them as "amorphous" linear dielectrics where a discontinuity of polarization across the grain boundaries can develop.

4.3.1. Effect of grain orientations. In order to evaluate only the effects of grain orientation, we analyse the configuration depicted in figure 15(a), where the specimen of size $L=H=100 \mathrm{~nm}$ contains now 12 grains randomly oriented with boundaries of zero thickness. More precisely, the grain boundaries are simply 1D hypersurfaces where the crystal axes change orientation, but besides this geometrical effect they are not equipped with any additional physical properties.

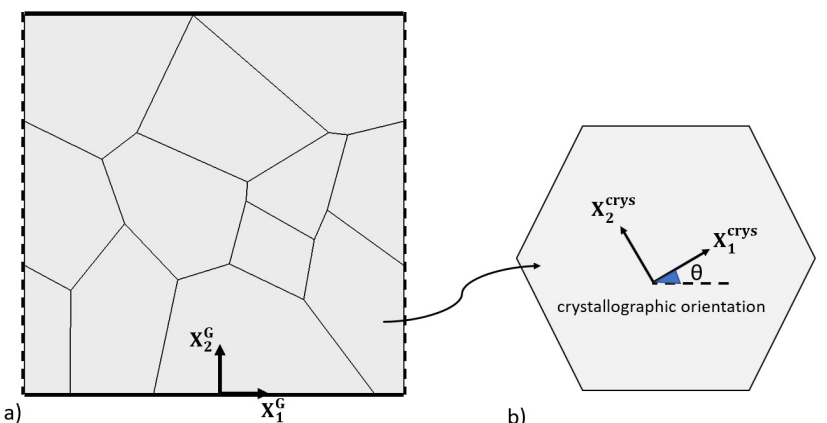

Figure 15: a) Polycrystalline sample and b) schematic crystallographic orientation of the grains.

The energy density introduced in section 2 has been defined for a single grain. In the presence of multiple grains, the free energy is expressed in the local reference system of each grain, which is described by three Euler angles, while the polarization, the strain and the electric field are backrotated to the global system by means of the rotation tensor $\mathbf{R}$ as $\mathbf{P}=$ $\mathbf{R} \cdot \mathbf{P}^{G}, \boldsymbol{\varepsilon}=\mathbf{R}^{\mathrm{T}} \cdot \boldsymbol{\varepsilon}^{G} \cdot \mathbf{R}$ and $\mathbf{E}=\mathbf{R} \cdot \mathbf{E}^{G}[49,72,51]$ where the superscript $G$ denotes the global fields. In the $2 \mathrm{D}$ case, the crystallographic orientation of each grain is only allowed around the out-of-plane axis by an angle $0 \leq \theta \leq \pi$ (see figure 15(b)). The rotation tensor is hence associated to the matrix

$[\mathbf{R}]=\left[\begin{array}{cc}\cos \theta & \sin \theta \\ -\sin \theta & \cos \theta\end{array}\right]$

Figure 16 shows the hysteresis loop and the domain evolution for the simulated polycrystalline ferroelectric. The boundary conditions and the simulation procedure are the same described previously. From the domain patterns, it is possible to observe that the polarization evolves continuously across the grains, though the orientation of the grains affects this evolution and makes the homogeneous state almost impossible to reach. It can be also remarked that, from the macroscopic point of view, the shape of the hysteresis loop is similar to the case of the perfect single crystal. However, the coercive field value $E_{C 180}$ is one order of magnitude smaller for the polycrystalline material. This difference is due to the fact that domain switching is easier when the crystallographic axes in the grains deviate from the direction of the applied electric field.

4.3.2. Effect of grain boundaries. For a complete description of the polycrystalline ferroelectric, we consider also the presence of amorphous grain boundaries with finite dimensions. Here, the polarization vector does not evolve according to the TDGL equation, but is assumed equal to

$\mathbf{P}=\kappa_{0}\left(\kappa_{r}-1\right) \mathbf{E}$ 
where $\kappa_{r}$ is the relative permittivity. Consequently, the dielectric displacement becomes

$\mathbf{D}=\kappa_{0} \mathbf{E}+\mathbf{P}=\kappa_{0} \kappa_{r} \mathbf{E}$

It is worth stressing that this approach apparently contrasts with $[50,51]$ where $\mathbf{P}=\mathbf{0}$ in the grain boundaries. However the difference is due to the fact that here we consider as order parameter of the PFM the total polarization and not only the spontaneous polarization (see the comments in section 2.1).

Let us now focus on the polycrystalline ferroelectric specimen in figure 17, which now includes grain boundaries of thickness $d=1 \mathrm{~nm}$ and $\kappa_{r}=66[50,51]$.

The simulated hysteresis loop and the domain patterns are plotted in figure 18. The values of the obtained electric coercive fields are $E_{C 90}=0.6 \times 10^{7}$ and $E_{C 180}=1.3 \times 10^{7}$. The large decrement is attributed to the presence of the finite thickness linear dielectric grain boundaries with a comparably low permittivity, which weakens the interaction of polarization across them. They have a shielding effect allowing the grains to switch more independently, as shown in the domain evolution, in which a variety of vortex-type polarization patterns can be observed as discussed in $[73,74,75]$.

Each grain switches approximatively at its own coercive field intensity, according to its orientation with respect to external electric field. On the contrary, in the previous case, all grains switch at the same time due to polarization coupling across grain boundaries. When a positive electric field is applied, the domains switch partially in the vertical direction. However, even for large values of $E_{\text {app }}$, the local polarizations are not fully oriented in the imposed direction (2),(4) due to the random orientation of the grains. When $E_{\text {app }}=0$, the random distribution of the grains and the presence of grain boundaries induce a reduction of the macroscopic remnant polarization $P_{r}$, which is equal to $0.29 \mathrm{C} / \mathrm{m}^{2}$.

It should be noted that the shape of $P \mathrm{vs} E$ in figure 18 is in close accordance with experimental observations (see, e.g., $[18,17]$ ), validating qualitatively our representation of polycrystalline ferroelectrics.

\section{Conclusion}

Defects have an essential impact on the nucleation and evolution of domains in ferroelectric materials. In this way they strongly influence the overall properties of ferroelectric devices. It is the aim of this study to understand this effect. To this end, a fully coupled electromechanical phase-field model with polarization as the order parameter governed by the GinzburgLandau equation has been implemented in an in-house Finite Element code. The code has been verified against results in literature. As the material, LeadTitanate (PTO) and Lead-Zirconate/Titanate (PZT) were chosen with the material parameters taken from literature. It is known that the coercive field strength obtained from single domain phase-field simulations is far beyond what is observed in experiments with real devices.

To investigate the impact of defects on the overall ferroelectric hysteresis behavior of single crystals, three types of defects have been investigated: (1) holes of different shape, (2) charged point defects, (3) pinning of the polarization. Furthermore, two types of grain boundaries as a kind of defect in a polycrystal have been considered, namely either as a purely geometrical feature of change in crystal orientation or as an additional dielectric phase between ferroelectric grains. To study the effect of defects, a $2 \mathrm{D}$ volume element under plane strain condition and periodic boundary conditions at the sides was subjected to complete poling and repoling cycle by a vertical electric field. The finite element mesh was refined to a degree that no artificial mesh pinning of the domains could occur.

For the holes, we assumed for simplicity an impermeable boundary condition at their surface. This is known to be a reasonable approximation for flaws of non-infinite diameter. Because of the strong effect of this electric boundary condition, holes showed a severe influence on the domain configuration in their neighborhood, even without applied electric field. In addition, the overall coercive field strength of the volume element was significantly reduced, since the presence of such a defect makes easier domain nucleation possible.

A charged point defect leads to the nucleation of new domains in a single crystal even without any external excitation. Our study showed how the coercive field strength of the volume element for 90and 180-degree switching is gradually decreased as the amount of the charge is increased.

To simulate domain pinning, the polarization in a part in the center of the simulated single crystal was fixed. Due to the strong effect of polarization coupling the overall hysteresis was severely affected. A strictly pinched hysteresis occurred resembling the hysteresis shape in aged devices. This finding supports understandings that polarization pinning is a possible source for aging in ferroelectric devices.

Concerning polycrystals, we have simulated them first with the grain boundaries having the only property to be hypersurfaces separating areas of different orientation of the crystal axes in different grains. The resulting hysteresis of the volume element was of a more or less rectangular shape as in the single crystal, however the coercive field strength was reduced by an order of magnitude. The rectangular shape is due 
to the strong polarization coupling between grains in this case, which triggers switching in all grains once it has been initiated in the first grain which was easiest to switch.

Finally, thin additional purely dielectric phases were introduced at the grain boundaries. These grain boundary phases led to a certain shielding and, thus, a decoupling of the domains in neighboring grains. As a result, instead of the more or less simultaneous switching of the domains in all grains in the previous case, now the grains rather switched more independently one after the other. The switching of the domains in a grain now mainly depends on the orientation of the crystal axes of this grain with respect to the external electric field in the first place. As a result a more rounded hysteresis loop with many small switching steps and a small overall coercive field strength is observed which reflects in a realistic way experimental observations.

This study shows that understanding the properties of ferroelectric devices requires looking not only at crystallographic features but also at defects. When the crystallographic properties are known, phase-field modeling is an appropriate approach to simulate the effect of defects on the overall material properties as they are encountered in ferroelectric devices.

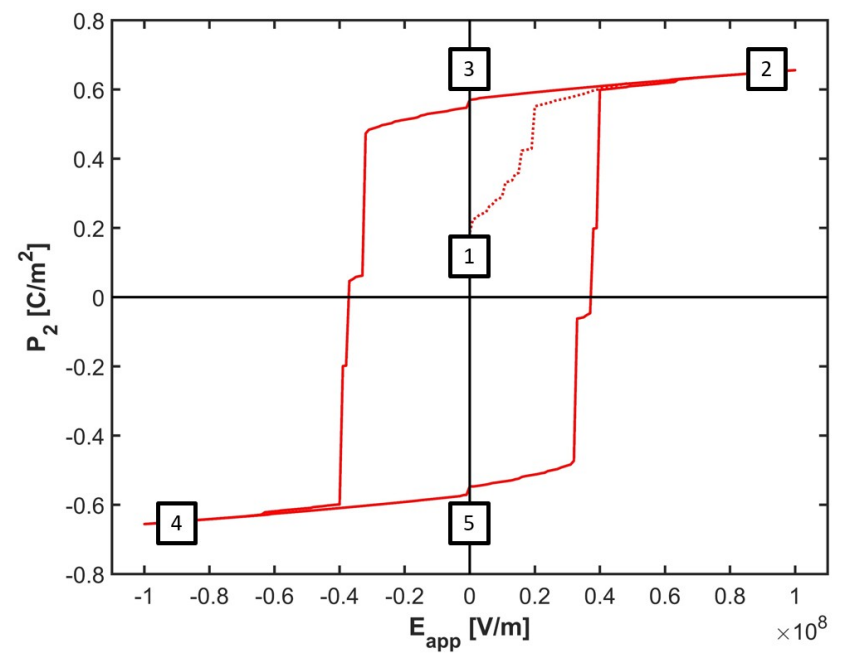

(a) Hysteresis loop PvsE
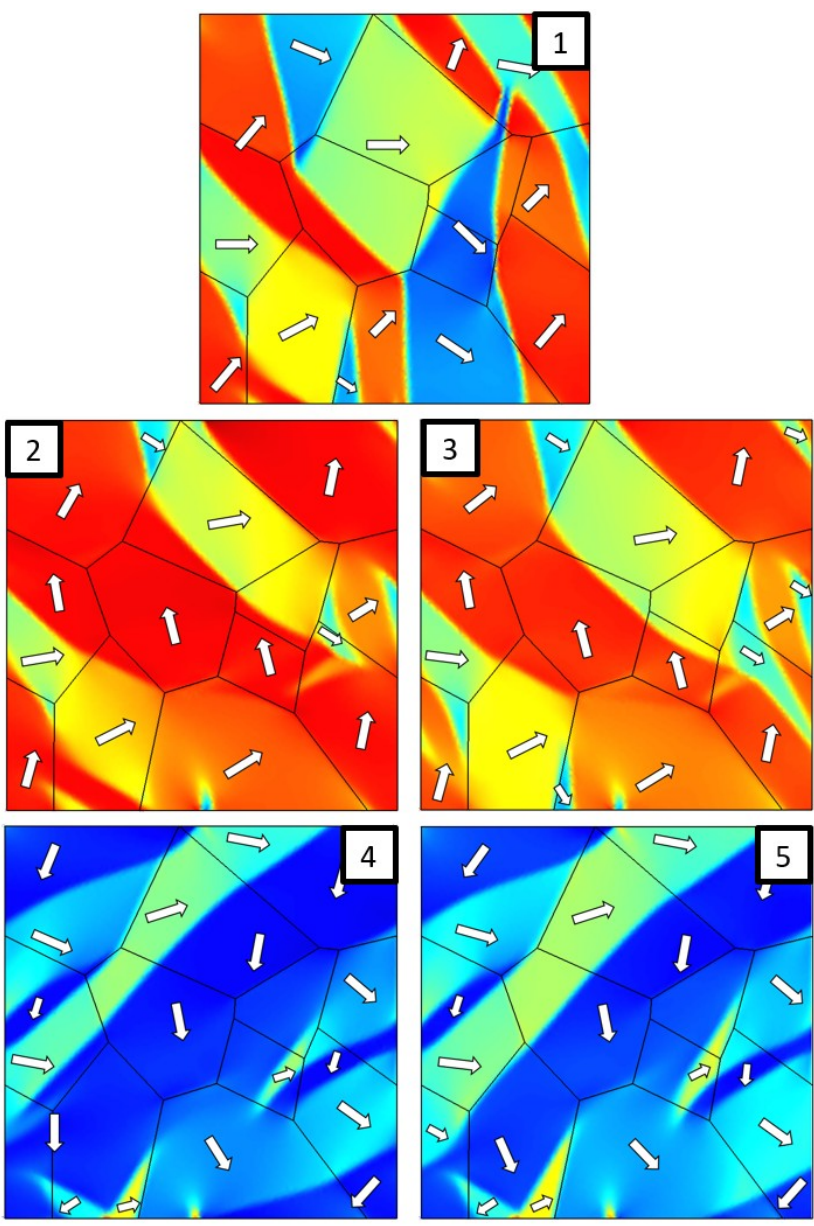

(b) Domain evolution

Figure 16: Polycrystalline ferroelectric with misoriented grains and zero thickness grain boundaries: a) hysteresis loop and b) contour plots for $P_{2}$. The arrows in the domains denote the orientation of polarization vector. 


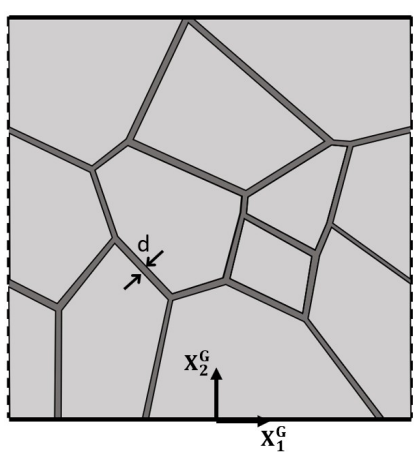

Figure 17: Polycrystalline ferroelectric with grain boundaries.

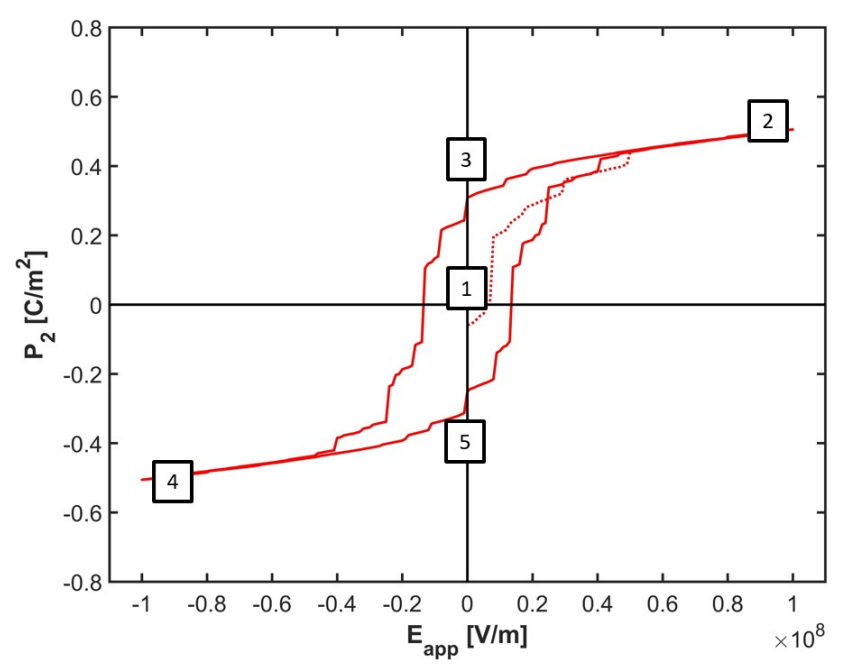

(a) Hysteresis loop PvsE
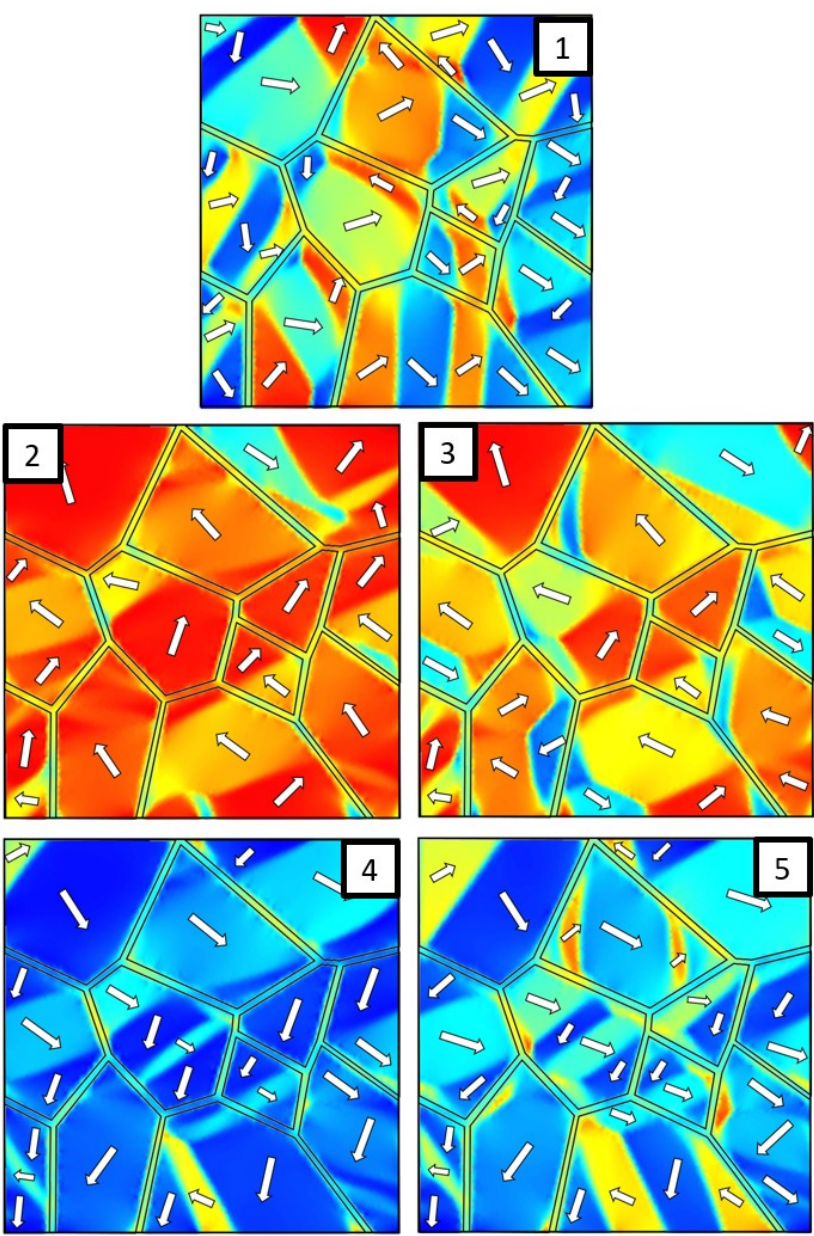

(b) Domain evolution

Figure 18: Polycrystalline ferroelectric with dielectric grain boundaries of thickness $1 \mathrm{~nm}$ and $\kappa_{r}=66$ : a) hysteresis loop and b) contour plots for $P_{2}$. The arrows in the domain evolution indicate the orientation of polarization vector. 


\section{Appendix A. Energy coefficients}

The material coefficients for $\mathrm{PbTiO}_{3}$ utilized in the simulations are taken from the work of Haun [76] and are listed in table A1. The spontaneous polarization magnitude $P^{s}=\left|\mathbf{P}^{s}\right|$ at room temperature is equal to $0.757 \mathrm{C} / \mathrm{m}^{2}$ and the normal spontaneous strain is: $\varepsilon_{\perp}=-0.0149$. Following previous works (see for instance [57,34]), the gradient coefficient $G_{11}$ is taken proportional to a reference value $G_{110}=1.73$. $10^{-10} \mathrm{C}^{-2} \mathrm{~m}^{3} \mathrm{~J}$. In particular, in our simulations we have chosen $G_{11}=0.6 G_{110}$. The corresponding wall thickness is about $1.5 \mathrm{~nm}$.

\begin{tabular}{|lcc|}
\hline Coefficient & Value & Unit \\
\hline$\alpha_{1}$ & -0.1725 & $(\mathrm{aJ})(\mathrm{nm})(\mathrm{aC})^{-2}$ \\
$\alpha_{11}$ & -0.073 & $(\mathrm{aJ})(\mathrm{nm})^{5}(\mathrm{aC})^{-4}$ \\
$\alpha_{12}$ & 0.75 & $(\mathrm{aJ})(\mathrm{nm})^{5}(\mathrm{aC})^{-4}$ \\
$\alpha_{111}$ & 0.26 & $(\mathrm{aJ})(\mathrm{nm})^{9}(\mathrm{aC})^{-6}$ \\
$\alpha_{112}$ & 0.61 & $(\mathrm{aJ})(\mathrm{nm})^{9}(\mathrm{aC})^{-6}$ \\
& & \\
$G_{11}$ & 0.1038 & $(\mathrm{aJ})(\mathrm{nm})^{3}(\mathrm{aC})^{-2}$ \\
& & \\
$C_{11}$ & 174 & $(\mathrm{aJ})(\mathrm{nm})^{-3}$ \\
$C_{12}$ & 79 & $(\mathrm{aJJ})(\mathrm{nm})^{-3}$ \\
$C_{44}$ & 111 & $(\mathrm{aJ})(\mathrm{nm})^{-3}$ \\
& & \\
$Q_{11}$ & 0.089 & $(\mathrm{~nm})^{4}(\mathrm{aC})^{-2}$ \\
$Q_{12}$ & -0.026 & $(\mathrm{~nm})^{4}(\mathrm{aC})^{-2}$ \\
$Q_{44}$ & 0.0338 & $(\mathrm{~nm})^{4}(\mathrm{aC})^{-2}$ \\
\hline
\end{tabular}

Table A1: Values of material coefficients for $\mathrm{PbTiO}_{3}$. 


\section{Appendix B. 2D description}

We rewrite constitutive equations (24)-(27) under the plane-strain assumption (38), and using Voigt's notation. The stress $\{\boldsymbol{\sigma}\}$ depends on elastic strains

$$
\left\{\begin{array}{l}
\sigma_{11} \\
\sigma_{22} \\
\sigma_{33} \\
\sigma_{12}
\end{array}\right\}=\left[\begin{array}{cccc}
C_{11} & C_{12} & C_{12} & 0 \\
C_{12} & C_{11} & C_{12} & 0 \\
C_{12} & C_{12} & C_{11} & 0 \\
0 & 0 & 0 & C_{44}
\end{array}\right]\left(\left\{\begin{array}{c}
\varepsilon_{11} \\
\varepsilon_{22} \\
\varepsilon_{33} \\
2 \varepsilon_{12}
\end{array}\right\}-\left\{\begin{array}{c}
\varepsilon_{11}^{s} \\
\varepsilon_{22}^{s} \\
\varepsilon_{33}^{s} \\
2 \varepsilon_{12}^{s}
\end{array}\right\}\right)
$$

where the spontaneous strain $\left\{\varepsilon^{s}\right\}$, according to (5), is a function of the polarization components:

$$
\left\{\begin{array}{l}
\sigma_{11} \\
\sigma_{22} \\
\sigma_{33} \\
\sigma_{12}
\end{array}\right\}=\left[\begin{array}{cccc}
C_{11} & C_{12} & C_{12} & 0 \\
C_{12} & C_{11} & C_{12} & 0 \\
C_{12} & C_{12} & C_{11} & 0 \\
0 & 0 & 0 & C_{44}
\end{array}\right]\left\{\left\{\begin{array}{c}
\varepsilon_{11} \\
\varepsilon_{22} \\
\varepsilon_{33} \\
2 \varepsilon_{12}
\end{array}\right\}-\left[\begin{array}{ccc}
Q_{11} P_{1} & Q_{12} P_{2} \\
Q_{12} P_{1} & Q_{11} P_{2} \\
Q_{12} P_{1} & Q_{12} P_{2} \\
Q_{44} P_{2} & Q_{44} P_{1}
\end{array}\right]\left\{\begin{array}{c}
P_{1} \\
P_{2}
\end{array}\right\}\right)
$$

We then write the expressions of the electric displacement vector $\{\mathbf{D}\}$ :

$\left\{\begin{array}{c}D_{1} \\ D_{2}\end{array}\right\}=\left[\begin{array}{cc}\kappa_{0} & 0 \\ 0 & \kappa_{0}\end{array}\right]\left\{\begin{array}{l}E_{1} \\ E_{2}\end{array}\right\}+\left\{\begin{array}{l}P_{1} \\ P_{2}\end{array}\right\}$

of the microstress $\{\boldsymbol{\xi}\}$ :

$$
\left\{\begin{array}{l}
\xi_{11} \\
\xi_{22} \\
\xi_{12} \\
\xi_{21}
\end{array}\right\}=\left[\begin{array}{cccc}
G_{11} & 0 & 0 & 0 \\
0 & G_{11} & 0 & 0 \\
0 & 0 & G_{11} & 0 \\
0 & 0 & 0 & G_{11}
\end{array}\right]\left\{\begin{array}{l}
P_{1,1} \\
P_{2,2} \\
P_{2,1} \\
P_{1,2}
\end{array}\right\}
$$

and finally of the microforce $\{\boldsymbol{\eta}\}$ :

$$
\begin{aligned}
\left\{\begin{array}{l}
\eta_{1} \\
\eta_{2}
\end{array}\right\}= & {\left[\begin{array}{ccc}
2 \alpha_{1}+4 \alpha_{11} P_{1}^{2}+2 \alpha_{12} P_{2}^{2} & 0 \\
+6 \alpha_{111} P_{1}^{4}+\alpha_{112}\left[4 P_{1}^{2} P_{2}^{2}+2 P_{2}^{4}\right] & 2 \alpha_{1}+4 \alpha_{11} P_{2}^{2}+2 \alpha_{12} P_{1}^{2} \\
0 & +6 \alpha_{111} P_{2}^{4}+\alpha_{112}\left[4 P_{1}^{2} P_{2}^{2}+2 P_{1}^{4}\right]
\end{array}\right]\left\{\begin{array}{c}
P_{1} \\
P_{2}
\end{array}\right\} } \\
& -2\left[\begin{array}{llll}
Q_{11} P_{1} & Q_{12} P_{1} & Q_{12} P_{1} & Q_{44} P_{2} \\
Q_{12} P_{2} & Q_{11} P_{2} & Q_{12} P_{2} & Q_{44} P_{1}
\end{array}\right]\left\{\begin{array}{l}
\sigma_{11} \\
\sigma_{22} \\
\sigma_{33} \\
\sigma_{12}
\end{array}\right\}-\left\{\begin{array}{c}
E_{1} \\
E_{2}
\end{array}\right\}
\end{aligned}
$$

where the matrix $[\boldsymbol{\alpha}(\mathbf{P})]$ contains the contribution of the Landau energy (2). 


\section{References}

[1] Walter Heywan, Karl Lubitz, and Wolfram Wersing, editors. Piezoelectricity: Evolution and Future of a Technology, volume 114. Springer Series in Materials Science, 2008

[2] ANSI/IEEE. An American National Standard IEEE Standard on Piezoelectricity. IEEE Standard, 176:1-74, 1988.

[3] B Jaffe, W R Cook, and H Jaffe. Piezoelectric Ceramics. London: Academic, 1971.

[4] Gene H. Haertling. Ferroelectric ceramics: History and technology. Journal of the American Ceramic Society, 82(4):797-818, 1999

[5] M.E. Lines and A.M. Glass. Priciples and Applications of Ferroelectrics and Related Materials. Claredon press, Oxford, 1977.

[6] Ernesto Suaste-Gomez. Piezoelectric Ceramics. Sciyo, 2010 .

[7] ANSI/IEEE. An american national standard IEEE standard definitions of terms associated with ferroelectric and related materials. IEEE transactions on ultrasonics, ferroelectrics, and frequency control, 50(12):1613-1646, 2003.

[8] N. Setter, D. Damjanovic, L. Eng, G. Fox, S. Gevorgian, S. Hong, A. Kingon, H. Kohlstedt, N. Y. Park, G. B. Stephenson, I. Stolitchnov, A. K. Taganstev, D. V. Taylor, T. Yamada, and S. Streiffer. Ferroelectric thin films: Review of materials, properties, and applications. Journal of Applied Physics, 100(5), 2006.

[9] P. Muralt. Ferroelectric thin films for micro-sensors and actuators: a review. Journal of Micromechanics and Microengineering, 10:136-146, 2000.

[10] Herman Wijshoff. The dynamics of the piezo inkjet printhead operation. Physics Reports, 491(4-5):77-177, 2010.

[11] G Massimino, A Colombo, L D Alessandro, F Procopio, and R Ardito. Multiphysics modelling and experimental validation of an air-coupled array of PMUTs with residual stresses. J. Micromech. Microeng., 28:054005, 2018.

[12] Sang-Gook Kim, Shashank Priya, and Isaku Kanno. Piezoelectric MEMS for energy harvesting. MRS Bulletin, 37(11):1039-1050, nov 2012.

[13] Alain B. Kounga, Torsten Granzow, Emil Aulbach, Manuel Hinterstein, and Jürgen Rödel. High-temperature poling of ferroelectrics. Journal of Applied Physics, 104(2):1-6, 2008.

[14] Ming Cheng Chure, Long Wu, Bing Huei Chen, Wei Ze $\mathrm{Li}$, Wei Kuo Liu, and Menq Jion Wu. Effect of poling conditions on the dielectric and piezoelectric characteristics of PZT ceramics. In Joint Conference of the 2009 Symposium on Piezoelectricity, Acoustic Waves, and Device Applications, SPAWDA 2009 and 2009 China Symposium on Frequency Control Technology, pages 317-320, 2009.

[15] Takeshi Kobayashi, Yasuhiro Suzuki, Natsumi Makimoto, Hiroshi Funakubo, and Ryutaro Maeda. Influence of pulse poling on the piezoelectric property of $\mathrm{Pb}(\mathrm{Zr} 0.52$, Ti0.48)O3 thin films. AIP Advances, 4(11):07, 2014.

[16] Z. X. Li, X. L. Liu, W. J. Chen, X. Y. Zhang, Ying Wang, W. M. Xiong, and Yue Zheng. Switchable diode effect in ferroelectric thin film: High dependence on poling process and temperature. AIP Advances, 4(12):0-11, 2014.

[17] Dragan Damjanovic. Hysteresis in piezoelectric and ferroelectric materials. In The Science of Hysteresis, volume 3, chapter 4, pages 337-465. Academic Press, 2005 .
[18] Gang Liu, Shujun Zhang, Wenhua Jiang, and Wenwu Cao. Losses in ferroelectric materials. Materials Science and Engineering R: Reports, 89:1-48, 2015.

[19] Venkatraman Gopalan, Volkmar Dierolf, and David A. Scrymgeour. Defect-Domain Wall Interactions in Trigonal Ferroelectrics. Annual Review of Materials Research, 37(1):449-489, 2007.

[20] Peng Gao, Christopher T. Nelson, Jacob R. Jokisaari, Seung Hyub Baek, Chung Wung Bark, Yi Zhang, Enge Wang, Darrell G. Schlom, Chang Beom Eom, and Xiaoqing Pan. Revealing the role of defects in ferroelectric switching with atomic resolution. Nature Communications, 2(1):591-596, 2011.

[21] Duo Liu. Microstructural Defects in Ferroelectrics and Their Scientific Implications. In M. Lallart, editor, Ferroelectrics - Characterization and Modeling, chapter 6, pages 97-114. InTech, 2011.

[22] Doru C. Lupascu. Fatigue in Ferroelectric Ceramic and Related Issues. Number 61. Springer Series in Materials Science, 2004.

[23] Yuri A. Genenko, Julia Glaum, Michael J. Hoffmann, and Karsten Albe. Mechanisms of aging and fatigue in ferroelectrics. Materials Science and Engineering B: Solid-State Materials for Advanced Technology, 192(C):52-82, 2015.

[24] M Kamlah. Ferroelectric and ferroelastic piezoceramics - modeling of electromechanical hysteresis phenomena. Continuum Mechanics and Thermodynamics, 13(4):219268, 2001.

[25] Chad M. Landis. Non-linear constitutive modeling of ferroelectrics. Current Opinion in Solid State and Materials Science, 8(1):59-69, 2004.

[26] B. Laskewitz and Marc Kamlah. Finite Element implementation of nonlinear constitutive models for piezoceramic materials. Journal of Mechanics of Materials and Structures, 5(1):19-45, 2010.

[27] Daining Fang, Faxin Li, Bin Liu, Yihui Zhang, Jiawang Hong, and Xianghua Guo. Advances in Developing Electromechanically Coupled Computational Methods for Piezoelectrics/Ferroelectrics at Multiscale. Applied Mechanics Reviews, 65(6):060802, 2013.

[28] Marc Kamlah and Charalampos Tsakmakis. Phenomenological modeling of the non-linear electro-mechanical coupling in ferroelectrics. International Journal of Solids and Structures, 36(5):669-695, 1999.

[29] V. Mehling, Ch Tsakmakis, and D. Gross. Phenomenological model for the macroscopical material behavior of ferroelectric ceramics. Journal of the Mechanics and Physics of Solids, 55(10):2106-2141, 2007.

[30] J. E. Huber. Micromechanical modelling of ferroelectrics. Current Opinion in Solid State and Materials Science, 9(3):100-106, 2005.

[31] W. Tang, D. N. Fang, and J. Y. Li. Two-scale micromechanics-based probabilistic modeling of domain switching in ferroelectric ceramics. Journal of the Mechanics and Physics of Solids, 57(10):1683-1701, 2009.

[32] R. Jayendiran, M. Ganapathi, and T. Ben Zineb. Finite element analysis of switching domains using ferroelectric and ferroelastic micromechanical model for single crystal piezoceramics. Ceramics International, 42(9):1122411238, 2016.

[33] L. Q. Chen. Phase -Field Models for Microstructure Evolution. Annual Review of Materials Research, 32(1):113-140, 2002.

[34] Jie Wang, San Qiang Shi, L. Q. Chen, Yulan Li, and Tong Yi Zhang. Phase-field simulations of ferroelectric/ferroelastic polarization switching. Acta Materialia, 52(3):749-764, 2004.

[35] W. Zhang and K. Bhattacharya. A computational model 
of ferroelectric domains. Part I: Model formulation and domain switching. Acta Materialia, 53(1):185-198, 2005.

[36] W. Zhang and K. Bhattacharya. A computational model of ferroelectric domains. Part II: Grain boundaries and defect pinning. Acta Materialia, 53(1):199-209, 2005.

[37] $\mathrm{Yu} \mathrm{Su}$ and C. M. Landis. Continuum thermodynamics of ferroelectric domain evolution: Theory, finite element implementation, and application to domain wall pinning. Journal of the Mechanics and Physics of Solids, $55(2): 280-305,2007$.

[38] D. Schrade, R. Müller, B. X. Xu, and D. Gross. Domain evolution in ferroelectric materials: A continuum phase field model and finite element implementation. Computer Methods in Applied Mechanics and Engineering, 196(41-44):4365-4374, 2007.

[39] Ronald E. Cohen. Origin of ferroelectricity in perovskite oxides. Nature, 358(6382):136-138, 1992.

[40] T Shimada, K Wakahara, Y Umeno, and $\mathrm{T}$ Kitamura. Shell model potential for PbTiO 3 and its applicability to surfaces and domain walls. Journal of Physics: Condensed Matter, 20(32):325225, 2008.

[41] Xiaowei Zeng and R. E. Cohen. Thermo-electromechanical response of a ferroelectric perovskite from molecular dynamics simulations. Applied Physics Letters, 99(14):97$100,2011$.

[42] Oliver Gindele, Anna Kimmel, Markys G. Cain, and Dorothy Duffy. Shell Model force field for Lead Zirconate Titanate $\mathrm{Pb}(\mathrm{Zr} 1 \times \mathrm{Ti} \times) \mathrm{O}$ 3. The Journal of Physical Chemistry C, 119(31):17784-17789, 2015.

[43] M. Graf, M. Sepliarsky, R. Machado, and M.G. Stachiotti. Dielectric and piezoelectric properties of $\mathrm{BiFeO} 3$ from molecular dynamics simulations. Solid State Communications, 218:10-13, 2015.

[44] Ruijuan Xu, Shi Liu, Ilya Grinberg, J Karthik, Anoop R Damodaran, Andrew M Rappe, and Lane W Martin. Ferroelectric polarization reversal via successive ferroelastic transitions. Nature materials, 14(1):79-86, 2015.

[45] Jun Wang, Yao Gen Shen, Fan Song, Fu Jiu Ke, Yi Long Bai, and Chun Sheng Lu. Effects of oxygen vacancies on polarization stability of barium titanate. Science China: Physics, Mechanics and Astronomy, 59(3):1-4, 2016.

[46] Benjamin Völker, P. Marton, C. Elässer, and Marc Kamlah. Multiscale modeling for ferroelectric materials: A transition from the atomic level to phase-field modeling. Continuum Mechanics and Thermodynamics, 23(5):435451, 2011.

[47] Benjamin Völker, Chad M Landis, and Marc Kamlah. Multiscale modeling for ferroelectric materials: identification of the phase-field model's free energy for PZT from atomistic simulations. Smart Materials and Structures, 21(3):35025, 2012.

[48] John W. Cahn and John E. Hilliard. Free energy of a nonuniform system. I. Interfacial free energy. The Journal of Chemical Physics, 28(2):258-267, 1958.

[49] S. Choudhury, Y. L. Li, C. E. Krill, and L. Q. Chen. Phasefield simulation of polarization switching and domain evolution in ferroelectric polycrystals. Acta Materialia, 53(20):5313-5321, 2005 .

[50] W. Shu, Jie Wang, and Tong Yi Zhang. Effect of grain boundary on the electromechanical response of ferroelectric polycrystals. Journal of Applied Physics, 112(6):0-16, 2012.

[51] Jie Wang, W. Shu, T. Shimada, T. Kitamura, and T. Y. Zhang. Role of grain orientation distribution in the ferroelectric and ferroelastic domain switching of ferroelectric polycrystals. Acta Materialia, 61(16):60376049, 2013.

[52] Jie Wang and Tong Yi Zhang. Size effects in epitaxial ferroelectric islands and thin films. Physical Review B Condensed Matter and Materials Physics, 73(14), 2006.
[53] Antonios Kontsos and C. M. Landis. Phase-Field Modeling of Domain Structure Energetics and Evolution in Ferroelectric Thin Films. Journal of Applied Mechanics, 77(4):041014, 2010.

[54] Y. L. Li, S. Y. Hu, Z. K. Liu, and L. Q. Chen. Phase-field model of domain structures in ferroelectric thin films. Applied Physics Letters, 78(24):3878-3880, 2001.

[55] Y. L. Li, S. Y. Hu, Z. K. Liu, and L. Q. Chen. Effect of electrical boundary conditions on ferroelectric domain structures in thin films. Applied Physics Letters, 81(3):427-429, 2002.

[56] Y. L. Li, S. Y. Hu, Z. K. Liu, and L. Q. Chen. Effect of substrate constraint on the stability and evolution of ferroelectric domain structures in thin films. Acta Materialia, 50(2):395-411, 2002.

[57] Y. L. Li, S. Y. Hu, and L. Q. Chen. Ferroelectric domain morphologies of (001) PbZr 1-xTi xO 3 epitaxial thin films. Journal of Applied Physics, 97(3):0-7, 2005.

[58] D. Schrade, R. Müller, B. X. Xu, and D. Gross. Domain wall pinning by point defects in ferroelectric materials. In Proc. SPIE 6526, Behavior and Mechanics of Multifunctional and Composite Materials 2007, volume 65260B, pages 1-8, 2007.

[59] Y. L. Li, S. Y. Hu, S. Choudhury, M. I. Baskes, A. Saxena, T. Lookman, Q. X. Jia, D. G. Schlom, and L. Q. Chen. Influence of interfacial dislocations on hysteresis loops of ferroelectric films. Journal of Applied Physics, 104(10):1-6, 2008

[60] Antonios Kontsos and Chad M. Landis. Computational modeling of domain wall interactions with dislocations in ferroelectric crystals. International Journal of Solids and Structures, 46(6):1491-1498, 2009.

[61] Jie Wang and M. Kamlah. Three-dimensional finite element modeling of polarization switching in a ferroelectric single domain with an impermeable notch. Smart Materials and Structures, 18(10):104008, 2009.

[62] Benjamin Völker and Marc Kamlah. Large-signal analysis of typical ferroelectric domain structures using phasefield modeling. Smart Materials and Structures, 21(5), 2012.

[63] A. F. Devonshire. Theory of ferroelectrics. Advances in Physics, 3(10):85-130, 1954.

[64] Jie Wang, W. Shu, H. Fang, and M. Kamlah. Phase field simulations of the poling process and nonlinear behavior of ferroelectric polycrystals with semiconducting grain boundaries. Smart Materials and Structures, 23(9):11, 2014.

[65] Wenwu Cao and L. E. Cross. Theory of tetragonal twin structures in ferroelectric perovskites with a first-order phase transition. Physical Review B, 44(1):5-12, 1991.

[66] Morton E. Gurtin. Generalized Cahn-Hilliard equations based on a microforce balance. Physica D, pages 178192, 1996.

[67] Alexander K. Tagantsev. Landau expansion for ferroelectrics: Which variable to use? Ferroelectrics, 375(1 PART 1):19-27, 2008.

[68] Ananya Renuka Balakrishna and John E. Huber. Nanoscale domain patterns and a concept for an energy harvester. Smart Materials and Structures, 25(10), 2016.

[69] I. Münch, A. Renuka Balakrishna, and J. E. Huber. Periodic boundary conditions for the simulation of 3D domain patterns in tetragonal ferroelectric material. Archive of Applied Mechanics, pages 1-18, 2018.

[70] Dayu Zhou, Marc Kamlah, and Dietrich Munz. Effects of uniaxial prestress on the ferroelectric hysteretic response of soft PZT. Journal of the European Ceramic Society, 25(4):425-432, 2005.

[71] M. Krauß and I. Münch. A selective enhanced FE-method for phase field modeling of ferroelectric materials. Computational Mechanics, 57(1):105-122, 2016. 
[72] S. Choudhury, Y. L. Li, C. E. Krill, and L. Q. Chen. Effect of grain orientation and grain size on ferroelectric domain switching and evolution: Phase field simulations. Acta Materialia, 55(4):1415-1426, 2007.

[73] Nina Balke, Benjamin Winchester, Wei Ren, Ying Hao Chu, Anna N. Morozovska, Eugene A. Eliseev, Mark Huijben, Rama K. Vasudevan, Petro Maksymovych, Jason Britson, Stephen Jesse, Igor Kornev, Ramamoorthy Ramesh, Laurent Bellaiche, Long Qing Chen, and Sergei V. Kalinin. Enhanced electric conductivity at ferroelectric vortex cores in BiFeO3. Nature Physics, 8(1):81-88, 2012.

[74] Ananya Renuka Balakrishna and John E. Huber. Scale effects and the formation of polarization vortices in tetragonal ferroelectrics. Applied Physics Letters, 106(9), 2015.

[75] Rama K. Vasudevan, Weida Wu, Jeffrey R. Guest, Arthur P. Baddorf, Anna N. Morozovska, Eugene A. Eliseev, Nina Balke, V. Nagarajan, Peter Maksymovych, and Sergei V. Kalinin. Domain wall conduction and polarization-mediated transport in ferroelectrics. Advanced Functional Materials, 23(20):2592-2616, 2013

[76] M. J. Haun, E. Furman, S. J. Jang, H. A. McKinstry, and L. E. Cross. Thermodynamic theory of PbTiO3. Journal of Applied Physics, 62(8):3331-3338, 1987. 